\title{
Nonlinear Exothermic Contributions to Radio-Frequency Bonding
}

\author{
of Adhesives ${ }^{1}$
}

\author{
H.T. Banks ${ }^{2} \quad$ S.R. Durso ${ }^{3} \quad$ M.A. Goodhart ${ }^{2} \quad$ K. Ito ${ }^{2}$
}

June 1, 1998

\footnotetext{
${ }^{1}$ Research supported in part by the US Air Force Office of Scientific Research under grants AFOSR F49620-95-1-0236, AFOSR F-49620-98-1-0180 and AFOSR F-49620-95-1-0375 and by the US Department of Education under grant P200A40730.

${ }^{2}$ Center for Research in Scientific Computation and Department of Mathematics, Box 8205, North Carolina State University, Raleigh, NC 27695-8205.

${ }^{3}$ Lord Corporation, Thomas Lord Research Center, 110 Lord Drive, P.O. Box 8012, Cary, NC 27512-8012
} 


\begin{abstract}
This work describes an effort to model the radio-frequency curing of epoxy adhesives in bonding of composites. We present a model based on the three-dimensional heat equation which includes nonlinear heat generating terms. A constructive proof of existence and uniqueness of solutions to our governing equations is given, providing a framework for the development of a computational method. Furthermore, using experimental data, we apply the numerical method to study the importance of the nonlinear contributions of an internal exothermic reaction which takes place as the adhesive cures.
\end{abstract}

Key words: radio-frequency electromagnetic bonding, nonlinear thermal processes, operator splitting, existence and uniqueness

AMS subject classifications: 35A40, 35K55, 65C20, 80-08 


\section{Introduction}

\subsection{Discussion of Physical Process}

Radio-frequency curing of adhesives is a commercially important process which is used in a number of practical settings. It is used in the fixation of prosthetic joints in some fields of medicine, the acceleration of adhesive setting in the woodworking industry, and the bonding of automobile parts in the automotive industry. In this work we focus on the use of radio-frequency bonding in the automotive industry. The use of non-metallic automotive exterior body panels has grown significantly over the last decade. The most common of these materials is sheet molding compound (SMC), a glass reinforced polyester which provides corrosion resistance, weight reductions, and complex shape molding capability. These parts are typically molded in two layers and adhesively bonded in sandwich fashion around their perimeters to form rigid structures.

The adhesive is commonly applied in a viscous liquid or paste form. Radio-frequency, or dielectric, heating is often used to accelerate the cure rate of the adhesive. In this application, the SMC/adhesive/SMC joint is placed between two electrodes (Figure 1). These electrodes then make contact with the joint, compressing it to the desired adhesive bondline thickness. A high voltage electric field, oscillating at approximately $30 \mathrm{MHz}$, then passes through the joint for a predetermined period of time at preset power levels, exciting polar or ionic species in the joint materials and generating heat. In comparison to common adhesives, the SMC is dielectrically relatively inactive. Significant heat can be generated within the adhesive, however, causing it to rapidly undergo a phase transition from liquid to solid (curing), and effectively bonding the two substrates to one another. This process, which can be closely simulated on a laboratory scale using a smaller version of the RF bonding equipment described above, provides us with a physically interesting problem. We 


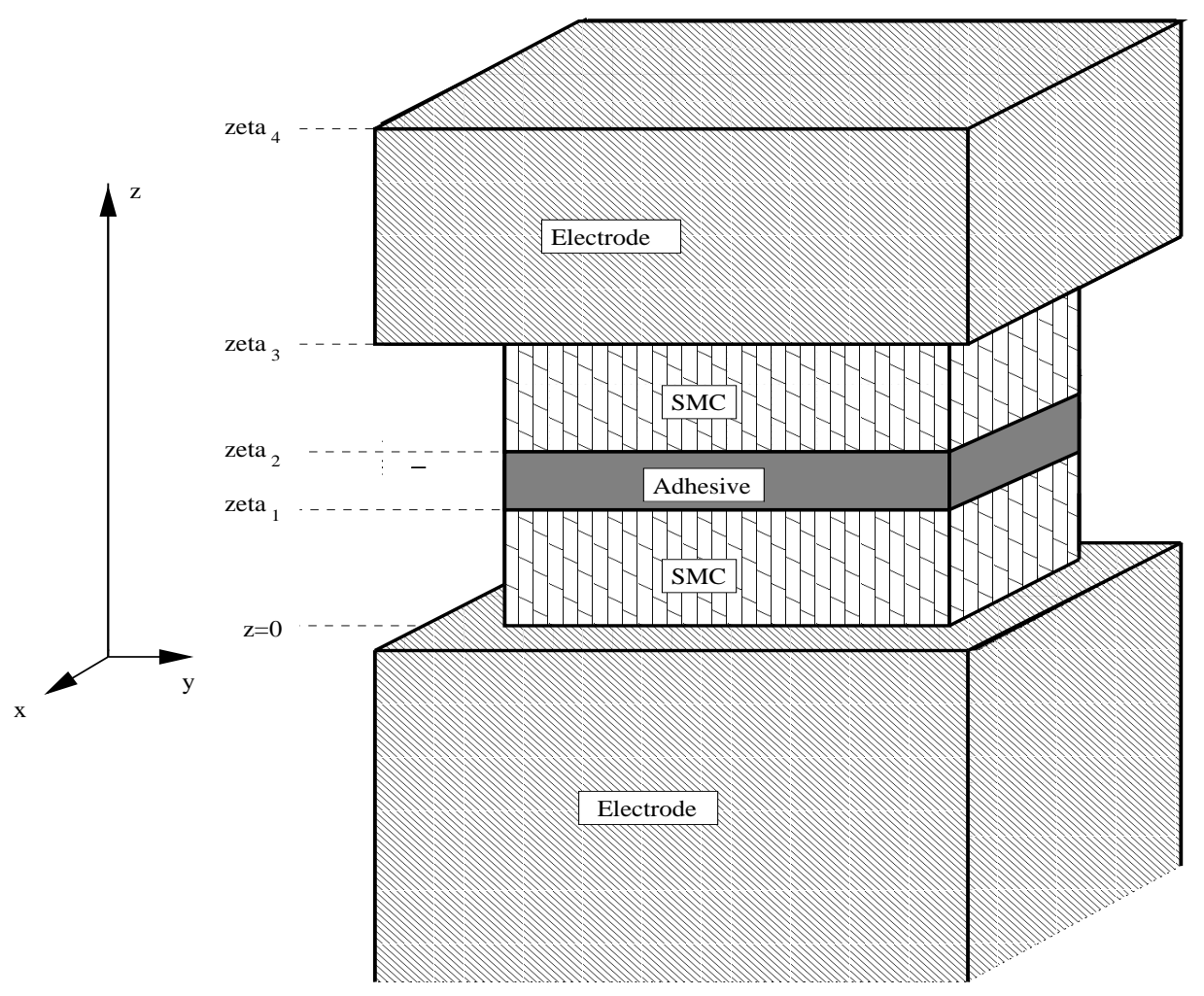

Figure 1: Diagram of SMC-adhesive-SMC joint.

must deal with thermally dependent nonlinearities arising from the radio-frequency field itself (i.e., temperature dependent input terms as well as conductivities), and complex internal phase transitions which are naturally parameterized by the degree of cure. This process thus provides us with a problem that is also mathematically interesting. It combines serious modeling issues, mathematical analysis, and computational methodology, while providing a foundation for necessary parameter estimation problems and nonlinear control methodology development.

Section 1.2 of this paper contains the formulation of our model of the physical process described above, taking into account the internal exothermic reaction as well as the heating contributed by the radio-frequency field. In Section 2 we provide the theoretical analysis of our problem, including 
a proof of existence and uniqueness which provides a foundation for the computational methods described in Section 3. Finally, in Section 4 we make use of the numerical methods described in Section 3 along with experimental data to investigate the appropriate form for the term used to approximate the exothermic reaction which takes place during the curing process.

\subsection{Mathematical Model}

In this section we formulate a heat transfer model for the physical experiment described in the introduction. We assume a physical sample as depicted in Figure 1 where the dimensions of the joint are described by $(0, a) \times(0, b) \times\left(0, \zeta_{4}\right)$. We model the heat transfer through the joint using the equation

$$
\rho c_{p} \frac{\partial T}{\partial t}=\nabla \cdot(\kappa \cdot \nabla T)+\dot{q}_{r f}+\dot{q}_{e x}
$$

Without the terms $\dot{q}_{r f}$ and $\dot{q}_{e x}$ this equation is simply the standard 3-dimensional heat equation where $\rho$ is mass density, $c_{p}$ is specific heat, $\rho c_{p}$ is volumetric heat capacity, and $\kappa$ is thermal conductivity. The terms $\dot{q}_{r f}$ and $\dot{q}_{e x}$, representing the rate of heating from conversion of electrical energy to molecular vibrational energy and the rate of heating due to some internal exothermic reaction, respectively, will be discussed later. We note that $\kappa, \rho$, and $c_{p}$ are material dependent parameters which, in general, will also depend on temperature and pressure. In addition, the values of each of these parameters may change in response to the phase transformation which occurs in the adhesive as the sample cures. For the sake of simplicity, however, we initially assumed that $\kappa, \rho$, and $c_{p}$ are constant within each material. Therefore, throughout this paper we define $\kappa, \rho$, and $c_{p}$ to be piecewise constant to account for the different materials in the system depicted in Figure 
1. Thus we have

$$
\kappa= \begin{cases}\kappa_{S M C} & 0 \leq z<\zeta_{1} \\ \kappa_{\text {adh }} & \zeta_{1} \leq z<\zeta_{2} \\ \kappa_{S M C} & \zeta_{2} \leq z<\zeta_{3} \\ \kappa_{\text {elec }} & \zeta_{3} \leq z<\zeta_{4}\end{cases}
$$

with similar definitions for $\rho$ and $c_{p}$.

In addition to (1), we must describe the initial and boundary conditions for the system. The bottom electrode contains heating and cooling fluids which enable the electrode to be held at a constant temperature $T_{1}$ for the duration of the experiment. In reality, the temperature of the electrode is initially higher than the temperature of the joint and hence there will be some heat transferred from the electrode to the joint. However, since the joint is heated very shortly after it is placed on the electrode, the amount of heat transferred is negligible. Similarly, there will be heat transferred from the joint to the electrode. Since the volume of the electrode is large compared to the volume of the joint and the electrode is held at a constant temperature, the heat transfer in this direction is also sufficiently small so as to be ignored. Hence, at the lower boundary where the sheet of SMC is in contact with the lower electrode $(z=0)$, the boundary condition is given by

$$
T(t, x, y, 0)=T_{b o t} .
$$

The upper electrode does not contain any temperature controlling fluids, and thus we can not easily hold the temperature constant. However, since the volume of the electrode is large compared to that of the SMC/adhesive/SMC joint, we assume that there is no heat flow at the top of the upper electrode $\left(z=\zeta_{4}\right)$, and hence the boundary condition is approximated by

$$
T\left(t, x, y, \zeta_{4}\right)=T_{t o p}
$$

The boundary terms given in (2) and (3) are essential boundary conditions which will be imposed 
on both strong and weak solutions.

At the interface where the top sheet of SMC contacts the upper electrode $\left(z=\zeta_{3}\right)$, there is a transfer of heat between the two materials. Hence, we have solid to solid conduction described by Fourier's Law which states that heat flux is given by $\kappa \frac{\partial T}{\partial z}[\mathrm{~B}, \mathrm{p} .9]$. If $\kappa_{\text {elec }}$ and $\kappa_{S M C}$ are the thermal conductivities of the electrode and the SMC, respectively, then continuity of flux requires

$$
\kappa_{\text {elec }} \frac{\partial T}{\partial z}\left(t, x, y, \zeta_{3}^{+}\right)=\kappa_{S M C} \frac{\partial T}{\partial z}\left(t, x, y, \zeta_{3}^{-}\right)
$$

with $T(t, x, y, z)$ continuous at $z=\zeta_{3}$ [ID, p.63]. Clearly, there will also be transfer of heat between the sheets of SMC and the adhesive layer. Thus, we must have continuity of flux at the interfaces between these materials and this yields

$$
\kappa_{a d h} \frac{\partial T}{\partial z}\left(t, x, y, \zeta_{i}^{ \pm}\right)=\kappa_{S M C} \frac{\partial T}{\partial z}\left(t, x, y, \zeta_{i}^{\mp}\right), \quad i=1,2
$$

The interface conditions given by (4) and (5) are natural conditions which will be automatically satisfied when we treat the system in weak or variational form (see Section 2).

At the sides of our system (i.e., surfaces defined by any one of the constraints $x=0, x=a, y=0$ or $y=b$ ) the joint is in contact with the air. As the temperature of the joint increases, the layer of air in contact with the joint will experience an increase in temperature and a decrease in density. Buoyancy forces induce a vertical motion, causing the warm air to be replaced with cooler ambient air. This causes a region called a boundary region to form through which temperature varies from the temperature of the surface of the joint to the ambient temperature $T_{\infty}$ [ID, p. 7-8]. This transfer of heat is called convective cooling and is described by Newton's Law of Cooling which can readily be expressed mathematically. For example, on the surface defined by $x=0$ it is given by

$$
-\kappa \frac{\partial T}{\partial n}(t, 0, y, z)=h\left(T(t, 0, y, z)-T_{\infty}\right), \quad 0<y<b, 0<z<\zeta_{4},
$$

where $h$ is the heat transfer coefficient of air [B, p. 23] and $\mathbf{n}$ is the outward normal to the surface 
$\left\{(0, y, z): 0<y<b, 0<z<\zeta_{4}\right\}$. This type of relationship also holds on the surfaces described respectively by $x=a, y=0$, and $y=b$. We note that (6) must take into account the fact that $\kappa$ is piecewise constant in the joint. The boundary terms described by (6) are also natural in nature, but these conditions involve nonhomogeneous terms which will appear in the weak formulation.

We now briefly discuss the term $\dot{q}_{r f}$ in (1) which is the rate of heat generated by conversion of electrical energy to molecular vibrational energy. It is reasonable to assume that the rate of heat generated in this manner is proportional to the applied voltage $U$. However, it might be expected that the "proportionality" between $\dot{q}_{r f}$ and the applied voltage will vary as the temperature of the system changes. This might be due, for example, to dependence of dielectric properties on temperature. Hence, as a first approximation we have a relationship of the form

$$
\dot{q}_{r f}=p(T) U
$$

The applied voltage is proportional to the electromagnetic field strength and hence (7) may be expressed in terms of the electromagnetic field strength using parameters which may be defined readily through experimentation. This term is commonly expressed in the literature ([M, p. 1271], [MC, p. 107], [BDGJ, p. 4] and references within) as

$$
\dot{q}_{r f}=\omega \varepsilon_{0} \varepsilon_{R}^{\prime} \tan \delta E_{R M S}^{2}
$$

where $\omega$ is the frequency of the applied electric field, $\varepsilon_{0}$ is the permittivity of free space, $\varepsilon_{R}^{\prime}$ is the relative dielectric constant, $\tan \delta$ is the dielectric loss tangent, and $E_{R M S}$ is the root mean square electric field strength. In [BDGJ] we offer a careful derivation of (8) and explain why this representation may not be a reasonable approximation under the conditions of our application. We then derive alternative expressions to (8) which might be more appropriate under these conditions.

The term $\dot{q}_{e x}$ represents the rate of heat generated by an internal exothermic reaction. The importance and form of this term are suggested in [BRA] wherein the authors present a model for 
exothermic polymerization in bone adhesives and also in $[\mathrm{MC}]$ where the authors discuss the role of exothermic heating in a method for selecting adhesives for curing in the automotive industry. The curing of the adhesive can be described in terms of a degree of cure $\alpha$, which is defined by

$$
\alpha=\left(\int_{0}^{t} S d t\right) / Q_{t o t}
$$

Here $S=\dot{q}_{e x}$ is the rate of heat generated per unit volume and $Q_{t o t}$ is the total amount of heat liberated per unit volume during the curing process. Although $Q_{\text {tot }}$ is generally temperature dependent, for many adhesives its variation about the mean is small enough that it is assumed to be constant [BRA, p. 253]. We shall show in Section 4 that such a model describes well the adhesive studied here. If we differentiate (9) with respect to time, we obtain a form for the term $\dot{q}_{e x}$ given by

$$
\dot{q}_{e x}=S=Q_{t o t} \frac{\partial \alpha}{\partial t}
$$

Similar to $[\mathrm{BRA}]$ and $[\mathrm{MC}]$, we require $\alpha(t)$ to satisfy an equation of the form

$$
\frac{\partial \alpha}{\partial t}=f(T) \Psi(\alpha)
$$

with initial condition

$$
\alpha(0)=\alpha_{0}
$$

Here the nonlinear terms $f$ and $\Psi$ are material dependent and must be determined from experimental data for a specific adhesive. The ordinary differential equation (10) is coupled with (1) and its boundary conditions (2)-(6) and must be solved as well. A method for solving these equations is presented in Section 3. The term $\dot{q}_{e x}$ and its importance with respect to our adhesive will be discussed in some detail in Section 4. 


\section{Existence and Uniqueness}

In this section we present existence and uniqueness results for a general nonlinear heat model motivated by the system of equations which govern the model proposed in Section 1.2. The analysis described within this section is based on an operator splitting method. A discussion of the background and theory for operator splitting is not given here, but may be found in literature, including $[\mathrm{LM}]$ and $[\mathrm{Y}]$. This use of this method to prove existence and uniqueness is advantageous since it also provides a foundation on which the numerical method detailed in Section 3 will be based.

Consider the heat generation and conduction equation of the form

$$
\rho c_{p} \frac{\partial}{\partial t} T=\nabla \cdot(\kappa \nabla T)+S_{c}
$$

in a general domain $\Omega \subset R^{3}$ with boundary $\partial \Omega=\Gamma_{1} \cup \Gamma_{2}$. We assume an initial condition $T(0, \mathbf{x})=$ $\mathrm{T}_{0}(\mathbf{x})$ and boundary conditions (fixed or Dirichlet on $\Gamma_{1}$, and Newton cooling on $\Gamma_{2}$ )

$$
T=g_{1} \text { on } \Gamma_{1} \text { and } \kappa \mathbf{n} \cdot \nabla \mathrm{T}=\mathrm{h}\left(g_{2}-\mathrm{T}\right) \text { on } \Gamma_{2} \text {. }
$$

We make the following assumptions on $g_{1}, g_{2}$.

(H1) The Dirichlet boundary term is given by $g_{1}=\operatorname{tr} g$ on $\Gamma_{1}$ for some $g \in H^{1}(\Omega)$ where $\operatorname{tr}$ is the trace operator.

(H2) The boundary term $g_{2}$ is in $L^{\infty}\left(\Gamma_{2}\right)$.

(H3) The boundary terms satisfy $g_{i}>0$ a.e. in $\Gamma_{i}$ for $i=1,2$.

We further assume that

(H4) The initial temperature $T_{0}$ satisfies $T_{0} \in L^{\infty}(\Omega)$. 
Here, the combined rate of heat generation $S_{c}$ per unit volume is given by

$$
S_{c}=Q_{t o t} \frac{\partial}{\partial t} \alpha+p(T) U
$$

The exothermic polymerization term can be modeled by

$$
\frac{\partial}{\partial t} \alpha=f(T) \Psi(\alpha), \quad \alpha(0, \mathbf{x})=\alpha_{0}(\mathbf{x})
$$

where we assume

(H5) The initial degree of cure satisfies $0 \leq \alpha_{0}(\mathrm{x}) \leq 1$ a.e. for $\mathrm{x} \in \Omega$.

The heat generation by RF-heating is modeled by $p(T) U$ where $U$ is the applied voltage. We assume that the rate functions $f$ and $p$ satisfy the following conditions.

(H6) The rate functions $f$ and $p$ are positive and non-decreasing.

(H7) The functions $f$ and $p$ are globally Lipschitz.

We assume a specific form for the kinetic law $\Psi(\alpha)$ which is given by

$$
\Psi(\alpha)=\psi(\alpha)(1-\alpha)
$$

We make the following assumptions about (15).

(H8) The function $\psi(\alpha)$ is a strictly increasing, Lipschitz continuous function with $\psi(0)=0$.

(H9) There exists a constant $\Psi_{\max }$ such that $\Psi(\alpha) \leq \Psi_{\max }$ on $[0,1]$.

In order to simplify our analysis, we also assume that

(H10) The mass density $\rho$, specific heat $c_{p}$, and thermal conductivity $\kappa$ are piecewise constant over fixed subdomains in $\Omega$.

For the sake of further simplicity in our discussions, without loss of generality we have tacitly 
assumed that the exothermic polymerizations are taking place throughout $\Omega$ (otherwise, we must distinguish subdomains without the polymerization equation (14)).

We use a variational formulation to define the solutions to (11)-(14). Let $H=L^{2}(\Omega)$ and $V=$ $\left\{\phi \in H^{1}(\Omega):\left.\phi\right|_{\Gamma_{1}}=0\right\}$. Then, $V \hookrightarrow H=H^{*} \hookrightarrow V^{*}$ defines a Gelfand triple. The weak form of (11) is given by

$$
\begin{aligned}
& \left\langle\rho c_{p} \frac{d}{d t} T(t), \phi\right\rangle_{V^{*}, V}+\langle\kappa \nabla T(t), \nabla \phi\rangle+\left\langle h\left(T(t)-g_{2}\right), \phi\right\rangle_{\Gamma_{2}} \\
& \quad=\left\langle Q_{t o t} f(T(t)) \Psi(\alpha(t))+U p(T(t)), \phi\right\rangle .
\end{aligned}
$$

Here $\langle\cdot, \cdot\rangle$ denotes the $L^{2}(\Omega)$ inner product, $\langle\cdot, \cdot\rangle_{V^{*}, V}$ is the usual duality product, and $\langle\cdot, \cdot\rangle_{\Gamma_{2}}$ denotes the inner product on $L^{2}\left(\Gamma_{2}\right)$. We establish the following existence and uniqueness result.

Theorem 2.1 Suppose that (H1)-(H10) are satisfied. Then there exists a unique solution $t \rightarrow(T(t), \alpha(t))$ to $(14)-(16)$ in $\left(L^{2}\left(0, \tau ; H^{1}(\Omega)\right) \cap H^{1}\left(0, \tau ; V^{*}\right) \cap L^{\infty}((0, \tau) \times \Omega)\right) \times H^{1}(0, \tau ; H)$.

Proof: We consider an operator-splitting method in a constructive format. Given the $k$-th timestep $\left(T^{k}, \alpha^{k}\right)$ at $t^{k}=k \Delta t$ we calculate $\left(T^{k+1}, \alpha^{k+1}\right)$ as follows. Solve

$$
\rho c_{p} \frac{T^{k+1}-T^{k}}{\Delta t}=\nabla \cdot\left(\kappa \nabla T^{k+1}\right)+Q_{t o t} f\left(T^{k}\right) \Psi\left(\alpha^{k}\right)+p\left(T^{k}\right) U
$$

with boundary conditions (12) for $T^{k+1}$, and then solve

$$
\frac{\partial}{\partial t} \alpha=f\left(T^{k+1}\right) \Psi(\alpha), \quad \alpha\left(\left(t^{k}\right)^{+}\right)=\alpha^{k}
$$

for $\alpha(t)$ and set $\alpha^{k+1}=\alpha\left(t^{k+1}\right)$.

(Existence): We prove the existence of a solution by showing in the following steps that the operatorsplitting method generates the sequence $\left\{\left(T^{k}, \alpha^{k}\right)\right\}$ which converges to a solution as $\Delta t \rightarrow 0^{+}$.

Step 1: (Well-posedness of (17)-(18)) Let $\Sigma=\left\{\alpha \in L^{2}(\Omega): 0 \leq \alpha(\mathbf{x}) \leq 1\right.$ a.e. $\left.\mathbf{x} \in \Omega\right\}$. First, we 
note that (18) is an ordinary differential equation if $\mathrm{x} \in \Omega$ is fixed and it follows from (15) under (H6)-(H9) that (18) has a unique solution $\alpha(\cdot, \mathbf{x}) \in \Sigma$ for given $T^{k+1} \in L^{\infty}(\Omega)$ and $\alpha^{k} \in \Sigma$.

The weak form of (17) is as follows. We have that $T^{k+1} \in V+g$ must satisfy

$$
\begin{aligned}
&\left\langle\rho c_{p}\right.\left.\frac{T^{k+1}-T^{k}}{\Delta t}, \phi\right\rangle+\left\langle\kappa \nabla T^{k+1}, \nabla \phi\right\rangle+\left\langle h\left(T^{k+1}-g_{2}\right), \phi\right\rangle_{\Gamma_{2}} \\
&=\left\langle Q_{t o t} \Psi\left(\alpha^{k}\right) f\left(T^{k}\right)+p\left(T^{k}\right) U, \phi\right\rangle
\end{aligned}
$$

for all $\phi \in V$. Since the sesquilinear form $\sigma_{1}$ on $V \times V$ defined by

$$
\sigma_{1}(\phi, \eta)=\frac{1}{\Delta t}\left\langle\rho c_{p} \phi, \eta\right\rangle+\langle\kappa \nabla \phi, \nabla \eta\rangle+\langle h \phi, \eta\rangle_{\Gamma_{2}}
$$

for $\phi, \eta \in V$ is bounded and $V$-elliptic, it follows from the Lax-Milgram theorem that there exists a unique $T^{k+1} \in V+g$ satisfying (19) for given $\alpha^{k}$ and $T^{k} \in V+g$. Next we establish an $L^{\infty}$-bound for $T^{k}$. Let $\theta^{k}=\sup _{\mathbf{x}} T^{k}(\mathbf{x})$ and $a_{1}=\theta^{k}+\frac{\Delta t}{c}\left(Q_{\text {tot }} \Psi_{\text {max }} f\left(\theta_{k}\right)+p\left(\theta^{k}\right) U\right)$, where $c=\inf _{\mathbf{x}} \rho(\mathbf{x}) \mathbf{c}_{\mathrm{p}}(\mathbf{x})$. Without loss of generality we can assume since $g_{2} \in L^{\infty}\left(\Gamma_{2}\right)$ that $\sup _{\mathbf{x} \in \Gamma_{2}} g_{2} \leq \theta^{k}$. Using $\phi \in V$ defined by $\phi(\mathbf{x})=\sup \left\{\mathrm{T}^{\mathrm{k}+1}(\mathbf{x})-\mathrm{a}_{1}, 0\right\}$ in $(19)$, we obtain

$$
\left\langle\rho c_{p}\left(T^{k+1}-a_{1}+\delta^{k}\right), \phi\right\rangle+\Delta t\left(\langle\kappa \nabla \phi, \nabla \phi\rangle+\left\langle h\left(\left(T^{k+1}-a_{1}\right)+\left(a_{1}-g_{2}\right)\right), \phi\right\rangle_{\Gamma_{2}}\right)=0
$$

where

$$
\delta^{k}=a_{1}-T^{k}-\frac{\Delta t}{\rho c_{p}}\left(Q_{t o t} \Psi\left(\alpha^{k}\right) f\left(T^{k}\right)+p\left(T^{k}\right) U\right) \geq 0 \quad \text { a.e. } \mathbf{x} \in \Omega .
$$

Thus, (20) implies

$$
\left\langle\rho c_{p} \phi, \phi\right\rangle+\Delta t\left(\langle\kappa \nabla \phi, \nabla \phi\rangle+\langle h \phi, \phi\rangle_{\Gamma_{2}}\right) \leq 0
$$

and thus $\phi=0$. Hence, $\theta^{k+1}=\sup _{\mathbf{x}} T^{k+1}(x)$ satisfies

$$
c \frac{\theta^{k+1}-\theta^{k}}{\Delta t} \leq Q_{t o t} \Psi_{\max } f\left(\theta^{k}\right)+p\left(\theta^{k}\right) U
$$

Since $f$ and $p$ are Lipschitz continuous, there exists $L_{f}$ and $L_{p}$ such that

$$
\frac{f(\theta)}{1+\theta} \leq L_{f} \quad \text { and } \quad \frac{p(\theta)}{1+\theta} \leq L_{p}
$$


Since $s \rightarrow \log (1+s)$ is concave, multiplying both sides of $(21)$ by $\left(1+\theta^{k}\right)^{-1}$ we obtain

$$
\frac{\log \left(1+\theta^{k+1}\right)-\log \left(1+\theta^{k}\right)}{\Delta t} \leq \frac{Q_{t o t} \Psi_{\max } L_{f}+U L_{p}}{c}=\hat{\omega}
$$

and hence we have

$$
1+\theta^{k} \leq e^{k \hat{\omega} \Delta t}\left(1+\theta^{0}\right)
$$

Let $T_{\min }=\inf _{\mathbf{x}} T_{0}(\mathbf{x})$ and assume without loss of generality that $g_{2} \geq T_{\min }$. Then, setting $\phi=\inf _{\mathbf{x}}\left\{T^{k+1}(\mathbf{x})-\mathrm{T}_{\min }, 0\right\}$ in (19) we can show that $\phi=0$ using arguments similar to those given above since $Q_{\text {tot }} f(T) \Psi(\alpha)+U p(T) \geq 0$ for $T>0$ and $\alpha \in[0,1]$. Thus, $T^{k} \geq T_{\min }$ for all $k$.

Therefore, the operator splitting method (17)-(18) defines a unique sequence $\left\{\left(T^{k}, \alpha^{k}\right)\right\}$ in $\left(H^{1}(\Omega) \cap\right.$ $\left.L^{\infty}(\Omega)\right) \times \Sigma$.

Step 2: (A priori estimate) Setting $\phi=T^{k+1}-g \in V$ in (19), we obtain

$$
\begin{aligned}
& \frac{1}{2}\left(\left|T^{k+1}-g\right|_{\tilde{H}}^{2}+\left|T^{k+1}-T^{k}\right|_{\tilde{H}}^{2}-\left|T^{k}-g\right|_{\tilde{H}}^{2}\right)+\frac{\Delta t}{2}\left(\left\langle\kappa \nabla T^{k+1}, \nabla T^{k+1}\right\rangle+\left\langle h T^{k+1}, T^{k+1}\right\rangle_{\Gamma_{2}}\right) \\
& \leq \Delta t\left(\text { const. }+Q_{\text {tot }} \Psi_{\max } f\left(\theta^{k}\right)+p\left(\theta^{k}\right) U\right)\left|T^{k}-g\right|_{H}
\end{aligned}
$$

where $\tilde{H}$ is $H$ equipped with the weighted norm $|\phi|_{\tilde{H}}^{2}=\left\langle\rho c_{p} \phi, \phi\right\rangle$ and const. depends on $g, g_{2}$. Summing up (23) with respect to $k$ with $m \Delta t \geq \tau$, we obtain

$$
\sum_{k=0}^{m}\left|T^{k+1}-T^{k}\right|_{H}^{2}+\Delta t\left|\nabla T^{k+1}\right|^{2} \leq M_{1}
$$

for some positive constant $M_{1}$ depending on $g, \theta^{0}, T^{0}$. If we let $\beta=\nabla \alpha$, then from (18)

$$
\frac{\partial}{\partial t} \beta=f\left(T^{k+1}\right) \Psi^{\prime}(\alpha) \beta+f^{\prime}\left(T^{k+1}\right) \Psi(\alpha)\left(\nabla T^{k+1}\right)
$$

Since $\left|\Psi^{\prime}(\alpha)\right| \leq 2 L_{\psi}$ on $[0,1]$ where $L_{\psi}$ is the Lipschitz constant for $\psi$, it follows that

$$
\left|\beta\left(t^{k+1}\right)\right|_{L^{2}}^{2} \leq e^{\left(2 M L_{\psi}+1\right)\left(t^{k+1}-t^{k}\right)}\left[\left(M \Psi_{\max }\right)^{2}\left|\nabla T^{k+1}\right|^{2} \Delta t+\left|\beta\left(t^{k}\right)\right|_{L^{2}}^{2}\right]
$$


where $\mathrm{M}$ is a constant bound for $\sup _{\mathrm{x}} f\left(T^{k}(\mathbf{x})\right)$ and $\sup _{\mathbf{x}} f^{\prime}\left(T^{k}(\mathbf{x})\right)$. Dividing by $c^{k+1}=e^{\left(2 M L_{\psi}+1\right) t^{k+1}}$ and summing, we obtain

$$
\left|\beta\left(t^{m+1}\right)\right|_{L^{2}}^{2} \leq \sum_{k=0}^{m} c^{m+1-k}\left(M \Psi_{\max }\right)^{2} \Delta t\left|\nabla T^{k+1}\right|^{2}+c^{m+1}\left|\beta\left(t^{0}\right)\right|_{L^{2}}^{2}
$$

It then follows from (24) that

$$
\left|\beta^{k}\right|_{L^{2}}^{2} \leq\left(M \Psi_{\max }\right)^{2} \epsilon^{\left(2 M L_{\psi}+1\right) t^{k}}\left(\sum_{k=0}^{m} \Delta t\left|\nabla T^{k+1}\right|^{2}+|\beta(0)|_{L^{2}}^{2}\right) \leq M_{2}
$$

for some constant $M_{2}$.

Step 3: (Convergence) Let us define the functions

$$
T_{\Delta t}^{(1)}(t)=T^{k+1} \quad \text { and } \quad T_{\Delta t}^{(2)}(t)=T^{k}+\frac{t-t_{k}}{\Delta t}\left(T^{k+1}-T^{k}\right) \quad \text { if } t \in\left[t_{k}, t_{k+1}\right),
$$

and

$$
\alpha_{\Delta t}^{(1)}(t)=\alpha^{k} \quad \text { and } \quad \alpha_{\Delta t}^{(2)}(t)=\alpha(t) \quad \text { if } t \in\left[t_{k}, t_{k+1}\right)
$$

where $\alpha(t)$ is the solution to (18). We argue that $\left(T_{\Delta t}(t), \alpha_{\Delta t}(t)\right)$ converges to $(T(t), \alpha(t))$, a solution to (11)-(14). Equations (17)-(18) can be equivalently written as

$$
\begin{aligned}
\left\langle\rho c_{p}\right. & \left.\frac{\partial}{\partial t} T_{\Delta t}^{(2)}(t), \phi\right\rangle+\left\langle\kappa \nabla T_{\Delta t}^{(1)}(t), \nabla \phi\right\rangle+\left\langle h\left(T_{\Delta t}^{(1)}(t)-g_{2}\right), \phi\right\rangle_{\Gamma_{2}} \\
& =\left\langle Q_{t o t} \Psi\left(\alpha_{\Delta t}^{(1)}(t)\right) f\left(T_{\Delta t}^{(1)}(t-\Delta t)\right)+p\left(T_{\Delta t}^{(1)}(t-\Delta t)\right) U, \phi\right\rangle
\end{aligned}
$$

for $\phi \in V$ and

$$
\frac{\partial}{\partial t} \alpha_{\Delta t}^{(2)}(t)=f\left(T_{\Delta t}^{(1)}(t)\right) \Psi\left(\alpha_{\Delta t}^{(2)}(t)\right)
$$

a.e. for $t$ in $(0, \tau)$. Equivalently, we may write

$$
\begin{gathered}
\left\langle\rho c_{p} T_{\Delta t}^{(2)}(t)-T_{0}, \phi\right\rangle+\int_{0}^{t}\left\langle\kappa \nabla T_{\Delta t}^{(1)}(s), \nabla \phi\right\rangle+\left\langle h\left(T_{\Delta t}^{(1)}(s)-g_{2}\right), \phi\right\rangle_{\Gamma_{2}} d s \\
=\int_{0}^{t}\left\langle Q_{t o t} \Psi\left(\alpha_{\Delta t}^{(1)}(s)\right) f\left(T_{\Delta t}^{(1)}(s-\Delta t)\right)+p\left(T_{\Delta t}^{(1)}(s-\Delta t)\right) U, \phi\right\rangle d s
\end{gathered}
$$

for $\phi \in V$. It follows from (19) that for $t \in\left(t^{k}, t^{k+1}\right)$

$$
\left|\frac{d}{d t} T_{\Delta t}^{(2)}(t)\right|_{V^{*}}^{2}=\left|\frac{T^{k+1}-T^{k}}{\Delta t}\right|_{V^{*}}^{2} \leq c_{1}\left(\left|\nabla T^{k+1}\right|^{2}+\left|g_{2}\right|_{L^{2}\left(\Gamma_{2}\right)}^{2}\right)+c_{2}
$$


for some constants $c_{1}, c_{2}$ and thus from $(24), \int_{0}^{\tau}\left|\frac{d}{d t} T_{\Delta t}^{(2)}(t)\right|_{V^{*}}^{2} d t$ is uniformly bounded. Hence from $(24)-(25)$

$$
\begin{aligned}
& T_{\Delta t}^{(2)}(t) \text { is a bounded set in } L^{2}\left(0, T ; H^{1}(\Omega)\right) \cap H^{1}\left(0, T ; V^{*}\right) \\
& \alpha_{\Delta t}^{(2)}(t) \text { is a bounded set in } L^{2}\left(0, T ; H^{1}(\Omega)\right) \cap H^{1}(0, T ; H) .
\end{aligned}
$$

Since $H^{1}(\Omega)$ is embedded compactly into $H=L^{2}(\Omega)$ it follows from [CF, Lemma 8.4] that the sequence $\left\{\left(T_{\Delta t}^{(2)}(t), \alpha_{\Delta t}^{(2)}(t)\right)\right\}$ has a subsequence which converges strongly in $L^{2}(0, \tau ; H)$ and weakly in $L^{2}\left(0, \tau ; H^{1}(\Omega)\right)$ to the limit $(T(t), \alpha(t))$. From $(24)$

$$
\begin{aligned}
& \left|T_{\Delta t}^{(1)}(t)-T_{\Delta t}^{(2)}(t)\right|_{L^{2}(0, \tau ; H)}^{2}=\frac{\Delta t}{3} \sum_{k=1}^{m}\left|T^{k+1}-T^{k}\right|_{H}^{2} \rightarrow 0 \\
& \left|T_{\Delta t}^{(1)}(t-\Delta t)-T_{\Delta t}^{(2)}(t)\right|_{L^{2}(0, \tau ; H)}=\frac{\Delta t}{3} \sum_{k=1}^{m}\left|T^{k+1}-T^{k}\right|_{H}^{2} \rightarrow 0
\end{aligned}
$$

as $\Delta t \rightarrow 0^{+}$. Similarly, from $(27)$ we have

$$
\left|\alpha_{\Delta t}^{(1)}(t)-\alpha_{\Delta t}^{(2)}(t)\right|_{L^{2}(0, \tau ; H)} \leq \Delta t \sqrt{\tau} M \Psi_{\max } \rightarrow 0
$$

as $\Delta t \rightarrow 0^{+}$. It thus follows from (29)-(30) that we can choose a subsequence of $\left.\left\{T_{\Delta t}^{(1)}(t), \alpha_{\Delta t}^{(1)}(t)\right)\right\}$ (which will be denoted by the same sequence) that converges to to some $(T, \alpha)$ weakly in $L^{2}\left(0, \tau ; H^{1}(\Omega)\right)$ and strongly in $L^{2}(0, \tau ; H)$. Since any $L^{2}((0, \tau) \times \Omega)$-convergent sequence has an a.e. in $(t, \mathbf{x})$ pointwise convergent subsequence, without loss of generality we can assume that

$$
\left(T_{\Delta t}^{(1)}(t), \alpha_{\Delta t}^{(1)}(t)\right) \rightarrow(T(t), \alpha(t)) \quad \text { for } t \in[0, \tau] \backslash E, \text { strongly in } H,
$$

where $E$ has zero Lebesgue measure. Applying the Lebesgue dominated convergence theorem and letting $\Delta t \rightarrow 0^{+}$in $(27)-(28)$, we obtain

$$
\begin{aligned}
&\left\langle\rho c_{p}\right.\left.\left(T(t)-T_{0}\right), \phi\right\rangle+\int_{0}^{t}\langle\kappa \nabla T(s), \nabla \phi\rangle+\left\langle h\left(T(s)-g_{2}\right), \phi\right\rangle_{\Gamma_{2}} d s \\
&=\int_{0}^{t}\left\langle Q_{t o t} \Psi(\alpha(s)) f(T(s))+p(T(s)) U, \phi\right\rangle d s
\end{aligned}
$$

for $\phi \in V$ and

$$
\alpha(t)-\alpha_{0}=\int_{0}^{t} f(T(s)) \Psi(\alpha(s)) d s
$$


for $t \in[0, T] \backslash E$. Since the integrands in (31)-(32) are integrable, it follows that (31)-(32) hold for $t \in[0, \tau]$, i.e., $(T(t), \alpha(t))$ is a solution to $(14),(16)$. Therefore, since $L^{\infty}((0, \tau) \times \Omega)$ is weak star complete, $T \in L^{2}\left(0, \tau ; H^{1}(\Omega)\right) \cap H^{1}\left(0, \tau ; V^{*}\right) \cap L^{\infty}((0, \tau) \times \Omega)$ and $\alpha \in H^{1}(0, \tau ; H)$ with $\alpha(t) \in \Sigma$ is a solution to (11)-(14) in the sense of $(31)-(32)$. Since $L^{2}(0, \tau ; V) \cap H^{1}\left(0, \tau ; V^{*}\right)$ is continuously embedded into $C(0, \tau ; H)$, we also have $T \in C(0, \tau ; H)$ and hence $\alpha \in C^{1}(0, \tau ; H)$.

(Uniqueness): Finally, we discuss the uniqueness of the solution to (11)-(14). Let $\left(T_{i}, \alpha_{i}\right), i=1,2$ be solutions to $(31)-(32)$ in $\left(L^{2}\left(0, \tau ; H^{1}(\Omega)\right) \cap H^{1}\left(0, \tau ; V^{*}\right) \cap L^{\infty}((0, \tau) \times \Omega)\right) \times H^{1}(0, \tau ; H \cap \Sigma)$. Since $\Psi$ and $f$ are Lipschitz continuous, $\alpha_{i}(t) \in \Sigma$, and $T_{i} \in L^{\infty}((0, \tau) \times \Omega)$, it is not difficult to prove (using standard Gronwall-based arguments) that

$$
\left|\alpha_{1}(t)-\alpha_{2}(t)\right|_{H} \leq M_{3} \max _{s \in[0, t]}\left|T_{1}(s)-T_{2}(s)\right|_{H}
$$

for some constant $M_{3}$. If we define

$$
\sigma_{2}(\phi, \eta)=\langle\kappa \nabla \phi, \nabla \eta\rangle+\langle h \phi, \eta\rangle_{\Gamma_{2}}
$$

then from (16) (or equivalently (31)) we have

$$
\begin{aligned}
& \left\langle\rho c_{p} \frac{d}{d t}\left(T_{1}(t)-T_{2}(t)\right), \phi\right\rangle_{V^{*}, V}+\sigma_{2}\left(T_{1}(t)-T_{2}(t), \phi\right) \\
& \quad=\left\langle Q_{t o t}\left(f\left(T_{1}(t)\right) \Psi\left(\alpha_{1}(t)\right)-f\left(T_{2}(t)\right) \Psi\left(\alpha_{2}(t)\right)\right)+U\left(p\left(T_{1}(t)\right)-p\left(T_{2}(t)\right)\right), \phi\right\rangle
\end{aligned}
$$

for $\phi \in V$. For $\eta \in L^{2}(0, \tau ; V) \cap H^{1}\left(0, \tau ; V^{*}\right)$ we have

$$
\frac{d}{d t}|\eta(t)|_{H}^{2}=2\left\langle\frac{d}{d t} \eta(t), \eta(t)\right\rangle_{V^{*}, V}
$$

a.e. $t \in(0, \tau)$. Thus, setting $\phi=T_{1}(t)-T_{2}(t)$ in $(34)$ we obtain

$$
\begin{aligned}
& \frac{1}{2} \frac{d}{d t}\left|T_{1}(t)-T_{2}(t)\right|_{\tilde{H}}^{2}+\sigma_{2}\left(T_{1}(t)-T_{2}(t), T_{1}(t)-T_{2}(t)\right) \\
& \quad=\left\langle Q_{t o t}\left(f\left(T_{1}(t)\right) \psi\left(\alpha_{1}(t)\right)-f\left(T_{2}(t)\right) \Psi\left(\alpha_{2}(t)\right)\right)+U\left(p\left(T_{1}(t)\right)-p\left(T_{2}(t)\right)\right), T_{1}(t)-T_{2}(t)\right\rangle \\
& \quad \leq\left(Q_{t o t} M L_{\psi}\left|\alpha_{1}(t)-\alpha_{2}(t)\right|_{H}+\left(Q_{\text {tot }} \Psi_{\max } L_{f}+U L_{p}\right)\left|T_{1}(t)-T_{2}(t)\right|_{H}\right)\left|T_{1}(t)-T_{2}(t)\right|_{H} .
\end{aligned}
$$


Integrating (35) with respect to $t$ we obtain

$\left.\frac{1}{2}\left|T_{1}(t)-T_{2}(t)\right|_{\tilde{H}}^{2} \leq \int_{0}^{t}\left(Q_{t o t} \Psi_{\max } L_{f}+U L_{p}\right)\left|T_{1}(s)-T_{2}(s)\right|_{H}^{2}+Q_{t o t} M L_{\psi}\left|\alpha_{1}(s)-\alpha_{2}(s)\right|_{H}\left|T_{1}(s)-T_{2}(s)\right|_{H}\right) d s$.

Let $r(t)=\max _{s \in[0, t]}\left|T_{1}(s)-T_{2}(s)\right|_{H}^{2}$. Then, from (33), (36), and the equivalence of $\tilde{H}$ and $H$, we have

$$
r(t) \leq 2 k_{1} \int_{0}^{t}\left(Q_{t o t}\left(\Psi_{\max } L_{f}+M M_{3} L_{\psi}\right)+U L_{p}\right) r(s) d s
$$

for some constant $k_{1}>0$. By Gronwall's inequality, $r(t)=0$ on $[0, \tau]$ and thus $\left(T_{1}(t), \alpha_{1}(t)\right)=$ $\left(T_{2}(t), \alpha_{2}(t)\right), t \in[0, \tau]$. Hence, $(T(t), \alpha(t))$ defines a unique solution to (11)-(14).

\section{Numerical Approximations}

We consider the 3 -dimensional equation given by (11), (13) and (14) in the rectangular solid $\Omega=$ $(0, a) \times(0, b) \times\left(0, \zeta_{4}\right)$. We assume that this solid is a single layer of material where the boundary conditions are assumed to be

$$
\kappa \frac{\partial T}{\partial n}=h\left(T_{\infty}-T\right)
$$

at the side boundary surface and

$$
T=T_{\text {top }} \text { on the top surface }\left(z=\zeta_{4}\right) \text { and } T=T_{\text {bot }} \text { on the bottom surface }(z=0) \text {. }
$$

These equations can be efficiently integrated using a central difference approximation for the spatial discretization and the operator splitting or fractional time-step method for the time integration. In this section we present a sketch of our integration method. The fractional time-step method used 
in our computations is given by

$$
\begin{aligned}
& \rho c_{p} \frac{T^{k+\frac{1}{2}}-T^{k}}{\Delta t}=\nabla \cdot\left(\kappa \nabla T^{k+\frac{1}{2}}\right) \\
& \frac{\alpha^{k+1}-\alpha^{k}}{\Delta t}=f\left(T^{k+\frac{1}{2}}\right) \Psi\left(\alpha^{k+1}\right) \\
& \rho c_{p} \frac{T^{k+1}-T^{k+\frac{1}{2}}}{\Delta t}=Q_{t o t} f\left(T^{k+1}\right) \Psi\left(\alpha^{k+1}\right)+p\left(T^{k+1}\right) U .
\end{aligned}
$$

The advantage of the fractional time-step method is that the three different process (i.e, the diffusion, kinetics for the $\alpha$, and heat generation) are separately integrated without losing the linear $O(\Delta t)$ accuracy of the implicit Euler method. The second and third equations of (39) are the implicit Euler method applied to the ordinary differential equations

$$
\begin{aligned}
& \frac{\partial}{\partial t} \alpha=f\left(T^{k+\frac{1}{2}}\right) \Psi(\alpha) \\
& \rho c_{p} \frac{\partial}{\partial t} T=Q_{t o t} f(T) \Psi\left(\alpha^{k+1}\right)+p(T) U
\end{aligned}
$$

at each point $(x, y, z) \in \Omega$ and thus can be carried out at each mesh point independently. The central difference approximation applied to the first equation of (39) can be written as the matrix Liapunov equation

$$
Q\left(\frac{\mathbf{T}^{\mathbf{k}+\frac{1}{2}}-\mathbf{T}^{\mathbf{k}}}{\Delta t}\right) \mathbf{Q}_{\mathbf{z}}+\mathrm{HT}^{\mathbf{k}+\frac{1}{2}} \mathbf{Q}_{\mathbf{z}}+\mathbf{Q T}^{\mathbf{k}+\frac{1}{2}} \mathbf{H}_{\mathbf{z}}=\mathbf{F}
$$

where the approximating temperature value $T_{i, j, l}^{k}$ at the 3 -dimensional uniformly distributed mesh point $\left(x_{i}, y_{j}, z_{l}\right) \in \Omega, 0 \leq i \leq m_{1}, 0 \leq j \leq m_{2}, 1 \leq l \leq m_{3}-1$ and at the time $k \Delta t$ is represented by the matrix $\mathbf{T}^{\mathbf{k}} \in \mathrm{R}^{\left(\mathrm{m}_{1}+1\right)\left(\mathrm{m}_{2}+1\right) \times\left(\mathrm{m}_{3}-1\right)}$. The $l$ th column $\mathbf{T}_{\cdot, \mathbf{l}}$ of $\mathbf{T}$ represents the values $T_{(i, j), l}, 0 \leq$ $i \leq m_{1}, 0 \leq j \leq m_{2}$, on the $z=z_{l}$ plane, ordered vectorwise, i.e., $T_{i\left(m_{1}+1\right)+j+1, l}=T_{i, j, l}$. The mass 
matrix $\mathbf{Q}_{\mathbf{z}}$ and stiffness matrix $\mathbf{H}_{\mathbf{z}}$ in the $z$-direction are given by

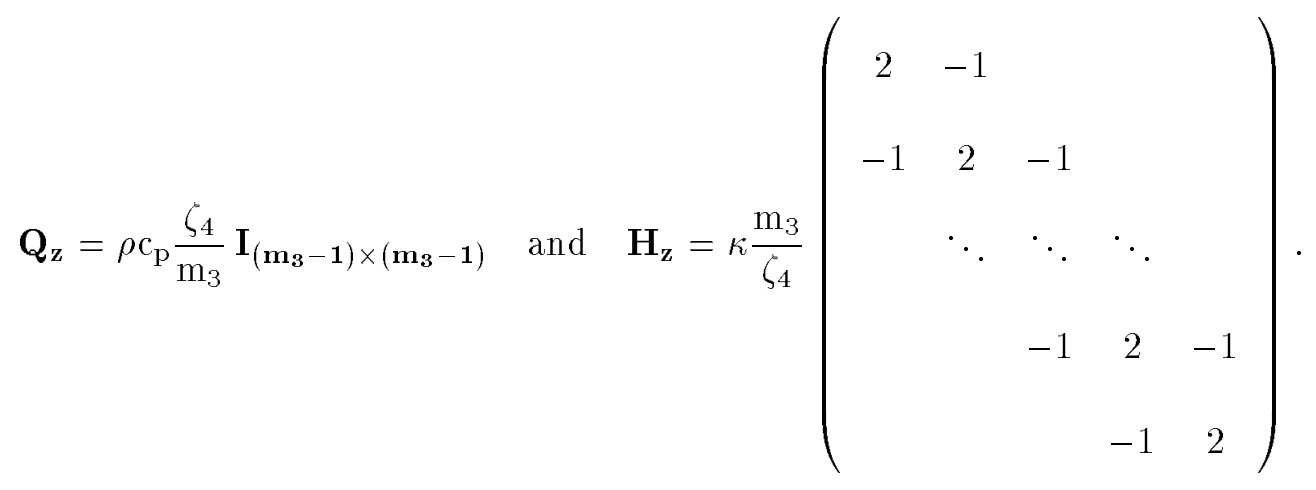

Given vector $\mathbf{v} \in \mathrm{R}^{\left(\mathrm{m}_{1}+1\right)\left(\mathrm{m}_{2}+1\right)}$, define the matrix $\mathbf{X} \in \mathrm{R}^{\left(\mathrm{m}_{1}+1\right) \times\left(\mathrm{m}_{2}+1\right)}$ by $X_{i, j}=v_{(i-1)\left(m_{1}+1\right)+j}$. We will denote this by $\mathbf{v}=\operatorname{vec}(\mathbf{X})$. Then $Q \mathbf{v}$ and $H \mathbf{v}$ are defined by

$$
Q \mathbf{v}=\operatorname{vec}\left(\mathbf{Q}_{\mathbf{x}} \mathbf{X} \mathbf{Q}_{\mathbf{y}}\right) \text { and } \mathrm{Hv}=\operatorname{vec}\left(\mathbf{H}_{\mathbf{x}} \mathbf{X} \mathbf{Q}_{\mathbf{y}}+\mathbf{Q}_{\mathbf{x}} \mathbf{X} \mathbf{H}_{\mathbf{y}}\right) \text {. }
$$

The mass matrices $\mathbf{Q}_{\mathbf{x}}, \mathbf{Q}_{\mathbf{y}}$ and stiffness matrices $\mathbf{H}_{\mathbf{x}}, \mathbf{H}_{\mathbf{y}}$ in the $x$ and $y$ direction, respectively are given by

$$
\mathbf{Q}_{\mathbf{x}}=\rho \mathbf{c}_{\mathrm{p}} \frac{\mathrm{a}}{\mathrm{m}_{1}} \mathbf{Q}_{\mathbf{0}}, \quad \mathbf{Q}_{\mathbf{y}}=\rho \mathbf{c}_{\mathrm{p}} \frac{\mathrm{b}}{\mathrm{m}_{2}} \mathbf{Q}_{\mathbf{0}} \quad \text { and } \quad \mathbf{H}_{\mathbf{x}}=\kappa \frac{\mathrm{m}_{1}}{\mathrm{a}} \mathbf{H}_{\mathbf{0}}, \quad \mathbf{H}_{\mathbf{y}}=\kappa \frac{\mathrm{m}_{2}}{\mathrm{~b}} \mathbf{H}_{\mathbf{0}}
$$

where $\mathbf{H}_{\mathbf{0}}$ is the tri-diagonal symmetric matrix of the form

$$
\mathbf{H}_{\mathbf{0}}=\left(\begin{array}{ccccc}
1+s & -1 & & & \\
-1 & 2 & -1 & & \\
& \ddots & \ddots & \ddots & \\
& & -1 & 2 & -1 \\
& & & -1 & 1+s
\end{array}\right)
$$

with $s=\frac{h}{\kappa} \frac{a}{m_{1}}$ for $\mathbf{H}_{\mathbf{x}}$ and $s=\frac{h}{\kappa} \frac{b}{m_{2}}$ for $\mathbf{H}_{\mathbf{y}}$, and the diagonal matrix $\mathbf{Q}_{\mathbf{0}}$ is defined by

$$
\mathbf{Q}_{0}=\operatorname{diag}\left(\frac{1}{2}, 1, \ldots, 1, \frac{1}{2}\right)
$$

The matrix $\mathbf{F} \in \mathrm{R}^{\left(\mathrm{m}_{1}+1\right)\left(\mathrm{m}_{2}+1\right) \times\left(\mathrm{m}_{3}-1\right)}$ represents the nonhomogeneities due to the nonhomogeneous boundary conditions (37)-(38). The matrix eigenvalue-eigenvector method for a solution to the 
Liapunov equation (40) is described in Appendix A. The node-wise nonlinear equations for the

second equation of (39) for $\alpha_{i, j, l}^{k+1}$ and the third equation for $T_{i, j, l}^{k+1}$ are solved by the Newton's method. The advantage of using the central difference is that the discrete maximum principle holds so that the positivity and possible monotonicity properties of solutions to the heat equation are maintained under approximations.

\section{Use of Numerical Methods to Study Exothermic Reaction}

In this section we discuss an application of the previously described theoretical and numerical ideas to the study of the exothermic reaction term. Originally, it was suggested that the exothermic source term $\dot{q}_{e x}$ in (1) was not very important. However, our simulations involving the heat equation without this term led to numerical results which were inconsistent with experimental observations. Thus, a major effort was focused on understanding the possible importance of exothermic input to overall heat generation. We found little in the literature to assist us in gaining insight into the importance of this term in our adhesive, but findings in an article on the use of adhesives in medical bonding [BRA] served as a starting point that held potential for us. Following this article, we designed experiments to possibly identify and quantify a form for the heat generated by the exothermic reaction. Using these experiments and the methods outlined in [BRA], we were able to formulate a model describing this exothermic heat contribution. At the same time we were developing an exothermic modeling term using [BRA] as an initiating point, findings by Malaczynski, et al. [MC] appeared which reinforced our belief that our own formulation was appropriate for the adhesive of interest to our investigations.

We discuss the formulation of the exothermic reaction model and discuss how this model can be included in the implementation of the numerical method. We compare two potential models which 
describe reasonably well this exothermic reaction. We also briefly discuss convergence of the finite difference method as $\Delta t \rightarrow 0$ and dependence on initial conditions. In the course of this discussion, we demonstrate the importance of including an exothermic source term in the overall model to more accurately approximate the physical behavior of our adhesive during the curing process.

\subsection{Formulation of Exothermic Model}

Recall from Section 1.2 that the rate of heat generated by the exothermic reaction $\left(\dot{q}_{\epsilon x}\right)$ may be described in terms of the degree of cure of the adhesive by

$$
\dot{q}_{e x}=S(T, \alpha)=Q_{t o t} \frac{\partial \alpha}{\partial t}
$$

where $\alpha(t)$ satisfies

$$
\frac{\partial \alpha}{\partial t}=f(T) \Psi(\alpha)
$$

The experiments mentioned above were designed to find the specific form of (42) for our adhesive. These experiments were carried out in the laboratory where the adhesive was isothermally heated in a digital scanning calorimeter (DSC). In an isothermal test, the sample is quickly heated (200 $\mathrm{C} / \mathrm{min}$ ) to the test temperature and held there for the duration of the experiment. Samples were maintained at $-50^{\circ} \mathrm{C}$ until the beginning of the test so that the rate of cure was minimized once the adhesive was mixed. Testing times were chosen so that the sample was fully cured by the end of the test. This experiment was performed at testing temperatures of $87.5^{\circ}, 100^{\circ}, 112.5^{\circ}, 125^{\circ}$, and $135.5^{\circ} \mathrm{C}$ (all within the range of normal curing temperatures for the adhesive) and yielded a power mass density $(\mathrm{W} / \mathrm{g}$ ) versus time (sec) curve for each temperature. 


\subsection{Converting Data to $S(\alpha)$ Curves}

Several steps were involved in converting the experimental data into the $S$ (rate of heat generated per unit volume) versus $\alpha$ (degree of cure) curves which were needed for the operator splitting step involving the exothermic reaction (39) in the numerical method. The portions of the data which represented initial heating of the sample from $-50^{\circ} \mathrm{C}$ to the target temperature were discarded since we were only concerned with the isothermal heating. Next, we multiplied the power mass density data by the volume mass density of the adhesive $\left(\rho=1.38 \mathrm{~g} / \mathrm{cm}^{3}\right)$ to obtain the $S\left(W / \mathrm{cm}^{3}\right)$ versus $t$ curves depicted in Figure 2. Using the relationship

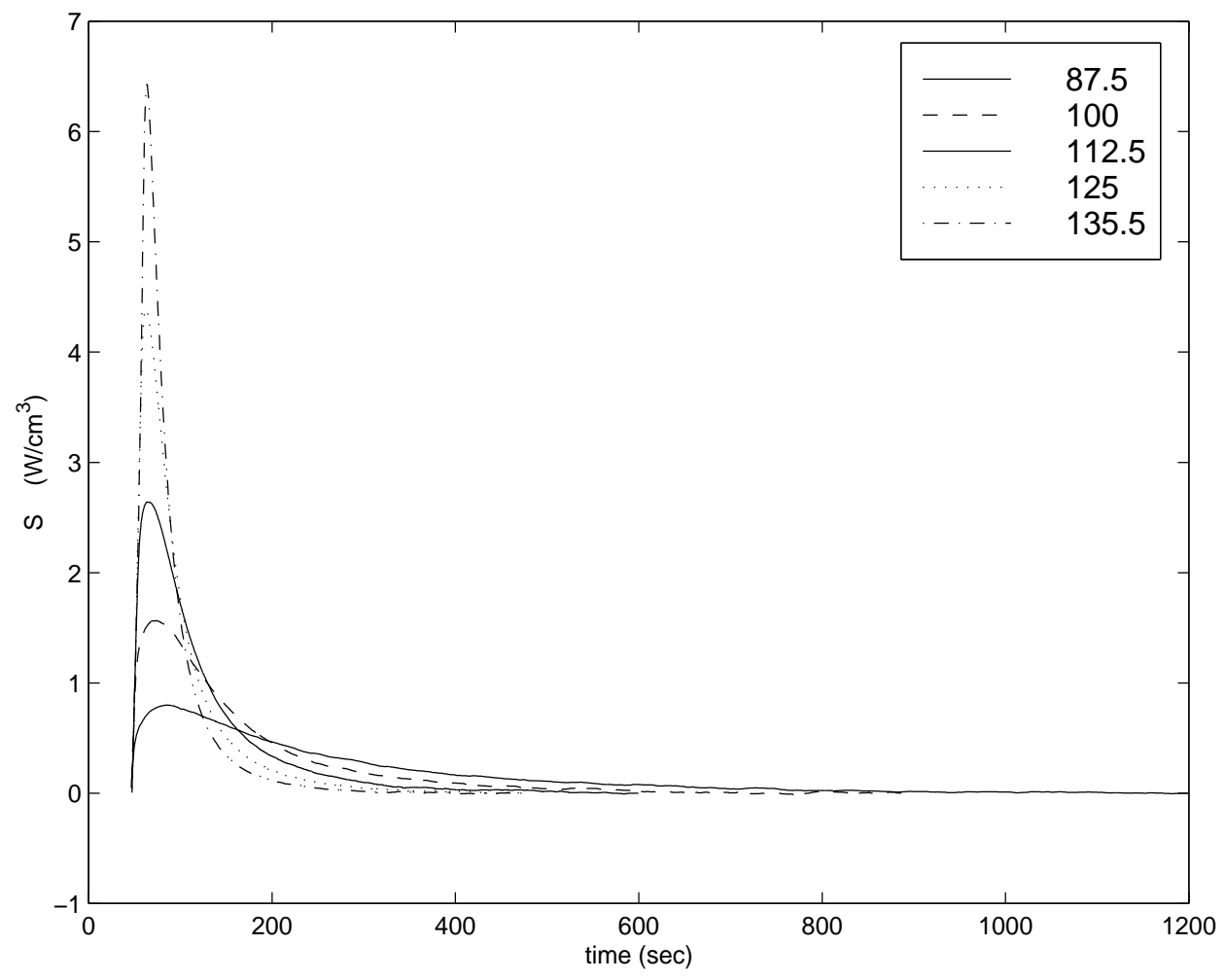

Figure 2: Rate of heat generated per unit volume $S$, versus time $t$, for various temperatures.

$$
\alpha(t)=\left(\int_{0}^{t} S(\tau) d \tau\right) / Q_{t o t}
$$


we converted each $S(t)$ curve into an $\alpha(t)$ curve by first applying the trapezoid rule with a relatively small step size to numerically integrate the data in each $S(t)$ curve and then dividing the resulting data by the total amount of heat liberated per unit volume $\left(Q_{t o t}\right)$. The value of $Q_{\text {tot }}$ was computed for each temperature by taking the supremum of the respective $S(t)$ curve. We used each $Q_{\text {tot }}$ with its respective curve to normalize the $\alpha(t)$ curves -see Figure 3 . However, since the values of $Q_{\text {tot }}$

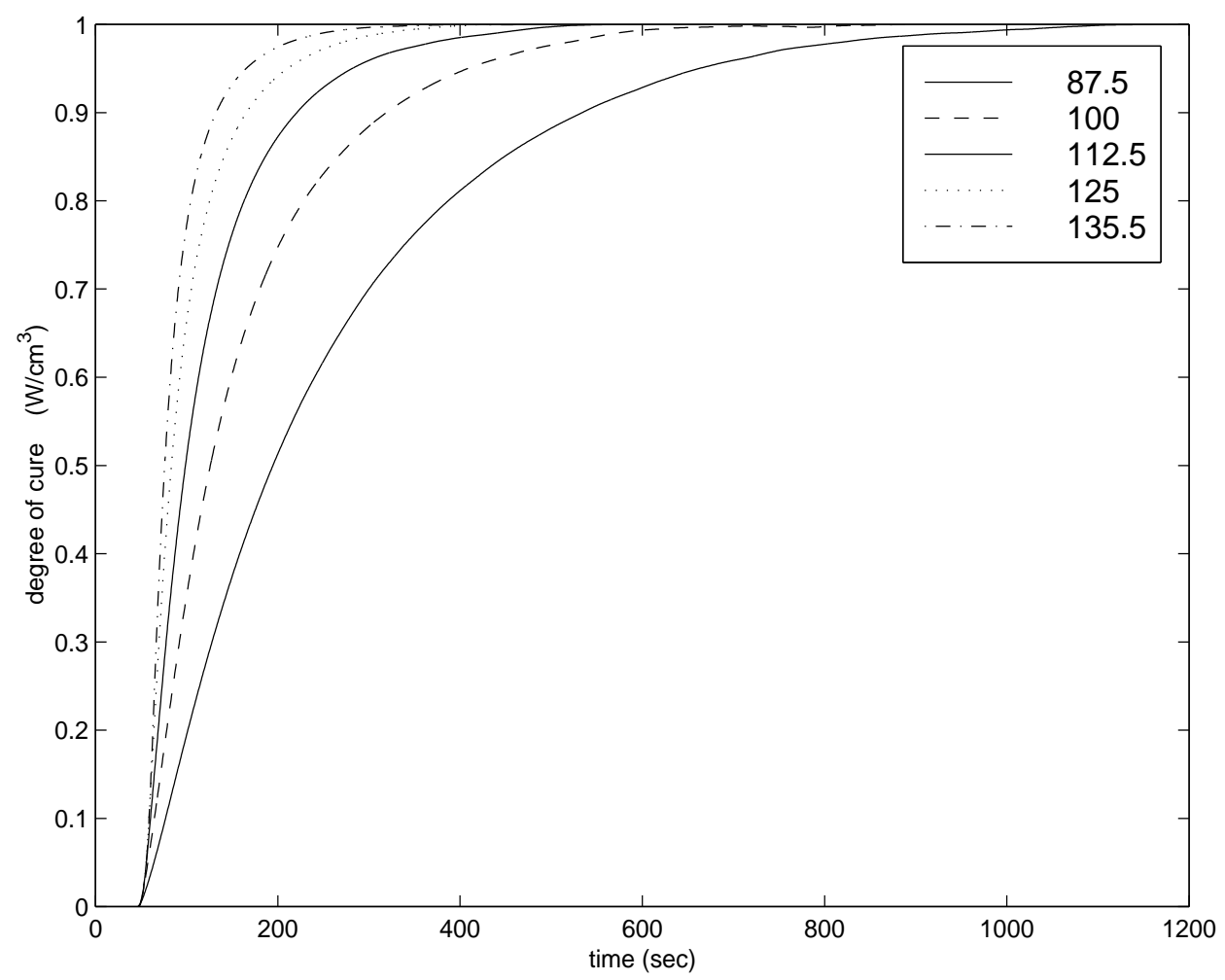

Figure 3: Degree of cure $\alpha$, versus time $t$, for various temperatures.

varied about the mean by less than $13 \%$, following [BRA] we assumed that $Q_{\text {tot }}$ was constant with respect to temperature to avoid any inconsistencies in our theory. Finally, we plotted the data points in $S$ against the points in $\alpha$ at their corresponding time values to get the final $S(\alpha)$ curves given in Figure 4. 


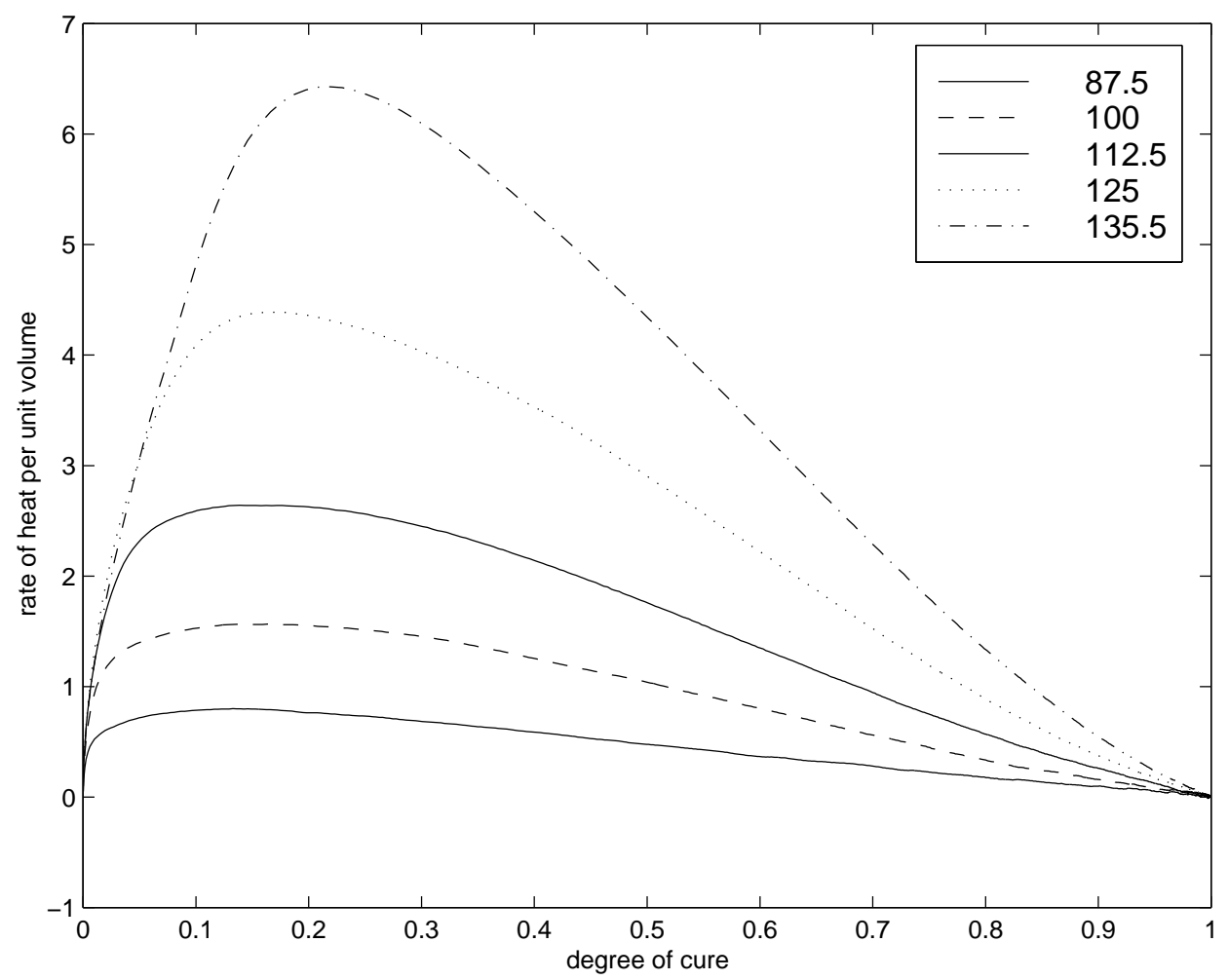

Figure 4: Rate of heat per unit volume $S$, versus degree of cure $\alpha$.

\subsection{Approximating the Curves}

The next step in developing the exothermic model was to approximate the $S(\alpha)$ curves by a function in the form of (42). We chose a function of the form

$$
\hat{S}(T, \alpha)=f(T)(1-\alpha) g(\alpha)
$$

based on the shape of the curves in Figure 4. We first attempted to fit the function

$$
\hat{S}(\alpha)=C(1-\alpha) \tan ^{-1}(k \alpha)
$$

to the curves at each temperature using a least squares approximation to find the values of $C$ and $k$. We first considered the case in which $k$ is constant. To obtain a constant $k$, we took an average of the values of $k$ found by the least squares method at the five temperatures and used the resulting 
$k=20$ in (44). We utilized two different methods to find the values of $C$ corresponding to $k=20$. First, we applied a least squares approximation to find the $C$ which minimized

$$
\sum_{i=1}^{N}\left|S_{i}-C\left(1-\alpha_{i}\right) \tan ^{-1}\left(20 \alpha_{i}\right)\right|^{2}
$$

at each temperature. Here $S_{i}$ and $\alpha_{i}$ represented the $i^{\text {th }}$ data points of the $S$ and $\alpha$ curves, respectively, at the chosen temperature. The second method involved matching the heights of the peaks of the $\hat{S}(\alpha)$ curves with the peaks of the $S(\alpha)$ curves for each temperature. Here we calculated the values of $C$ as

$$
C=\frac{\max _{\alpha}\{\hat{S}(\alpha)\}}{\max _{\alpha}\left\{(1-\alpha) \tan ^{-1}(20 \alpha)\right\}}
$$

which yielded a $C$ value at each of the five temperatures. Comparison of the resulting curves in Figure 5 demonstrated that the curves found using the peak matching method fit the original curves much better than those determined using the least squares method. Additionally, matching the heights of the peaks was important in this problem, and thus we chose the second method to find the values of $C$ in (44).

Finally, we interpolated the values of $C$ with a piecewise linear function $f(T)$ where

$$
f(T)= \begin{cases}.01 T & 0 \leq T<87.5 \\ .06 T-4.27 & 87.5 \leq T<100 \\ .08 T-6.61 & 100 \leq T<112.5 \\ .13 T-12.28 & 112.5 \leq T<125 \\ .18 T-18.65 & 125 \leq T .\end{cases}
$$

Hence, the final form of our initial approximation of $S(T, \alpha)$ with $(42)$ was

$$
\hat{S}(T, \alpha)=f(T)(1-\alpha) \tan ^{-1}(20 \alpha)
$$

with $f(T)$ given by (45). 


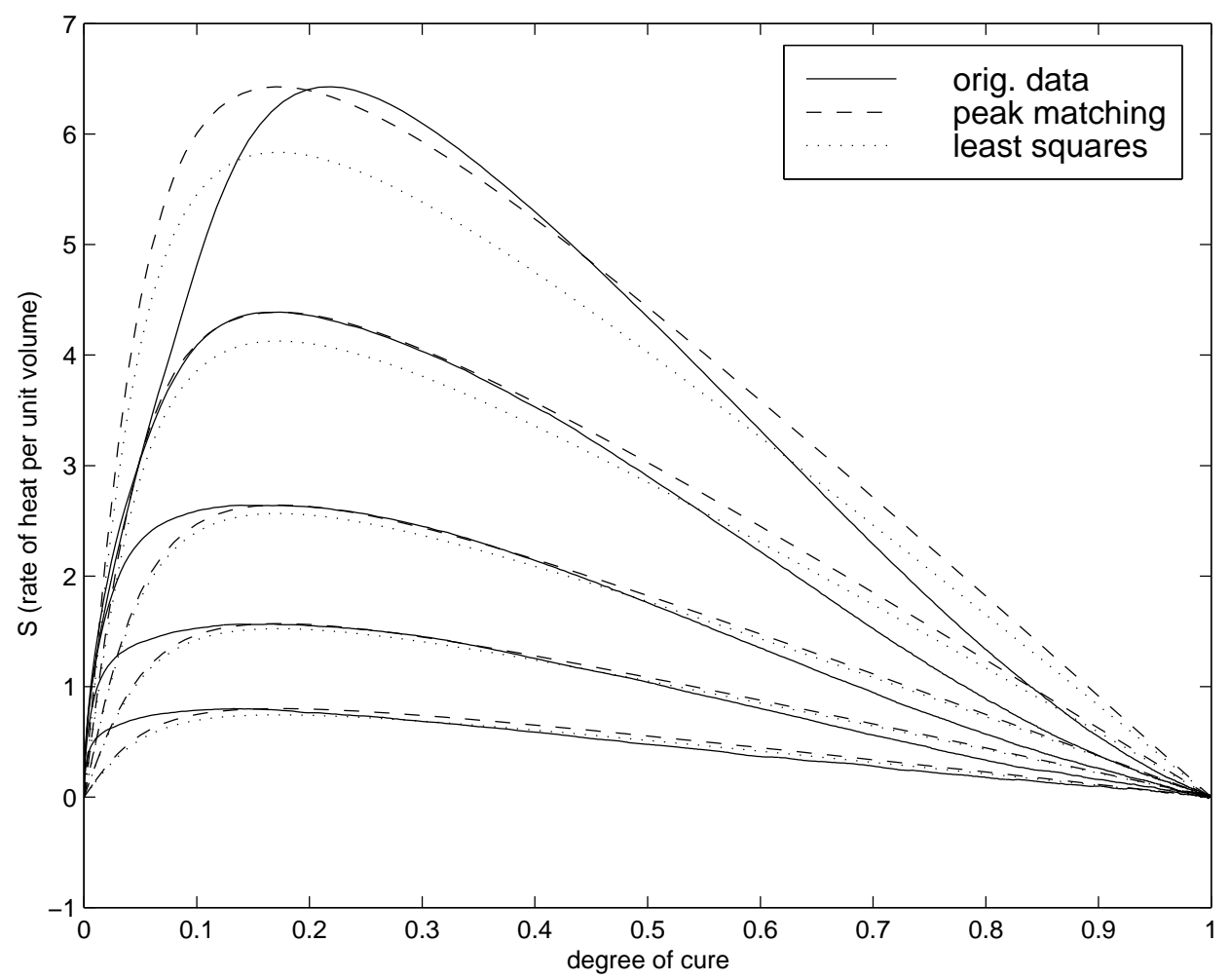

Figure 5: Comparison of peak matching method and least squares method to experimental data.

As a possible alternative we considered a function of the form

$$
\tilde{S}(T, \alpha)=f(T)(1-\alpha)\left(1-e^{-k \alpha}\right)
$$

as an approximation to the $S(\alpha)$ curves where $f(T)$ and $k$ were found using the methods detailed above. The resulting curves fit reasonably well, but did not match the peaks as nicely as the curves from (44), and hence were not used and are not shown here.

In addition, we explored the case where $k$ was allowed to vary with temperature in (44) by studying the function

$$
\bar{S}(T, \alpha)=f(T)(1-\alpha)^{m} \tan ^{-1}(K(T) \alpha)
$$

as an approximation to the $S(\alpha)$ curves. First, trial and error was used to determine that $m=1.25$ 
yielded curves whose shape closely matched that of the data curves when $K$ was allowed to vary. (Note: Using $(1-\alpha)^{1.25}$ in (44) and (47) did not make the approximations significantly better.) Again applying a nonlinear least squares method, we found the $C$ and $K$ which minimized

$$
\sum_{i=1}^{N}\left|S_{i}-C\left(1-\alpha_{i}\right)^{1.25} \tan ^{-1}\left(K \alpha_{i}\right)\right|^{2}
$$

at each temperature. Following the previously described method, we determined a piecewise linear function $f(T)$ which fit the values of $C$. A study of the values of $K$ showed that the values decreased almost linearly as temperature increased and hence we used a linear function to represent $K(T)$ for $0 \leq T \leq 135.5$. Also, since negative $K$ values resulted in undesirable behavior of the curves, $K(T)$ was held constant for $T>135.5$. Thus we set

$$
K(T)= \begin{cases}-.71931 T+107.6522 & 0 \leq T \leq 135.5 \\ 10.1857 & 135.5<T\end{cases}
$$

in (48). A comparison between (48) with $m=1.25$ and (44) depicted in Figure 6 showed that (48) fit the $S(\alpha)$ curves much more closely with respect to the shape of the curves and the placement of the peaks. However, implementing a function in the form of (48) resulted in added computations and a significant increase in computational time. We demonstrate in Section 4.4 .2 that using $\bar{S}$ and $\hat{S}$ yield very similar results in the final simulations with the code and therefore argue that it is sufficient to use (44) to approximate $S(\alpha)$. 


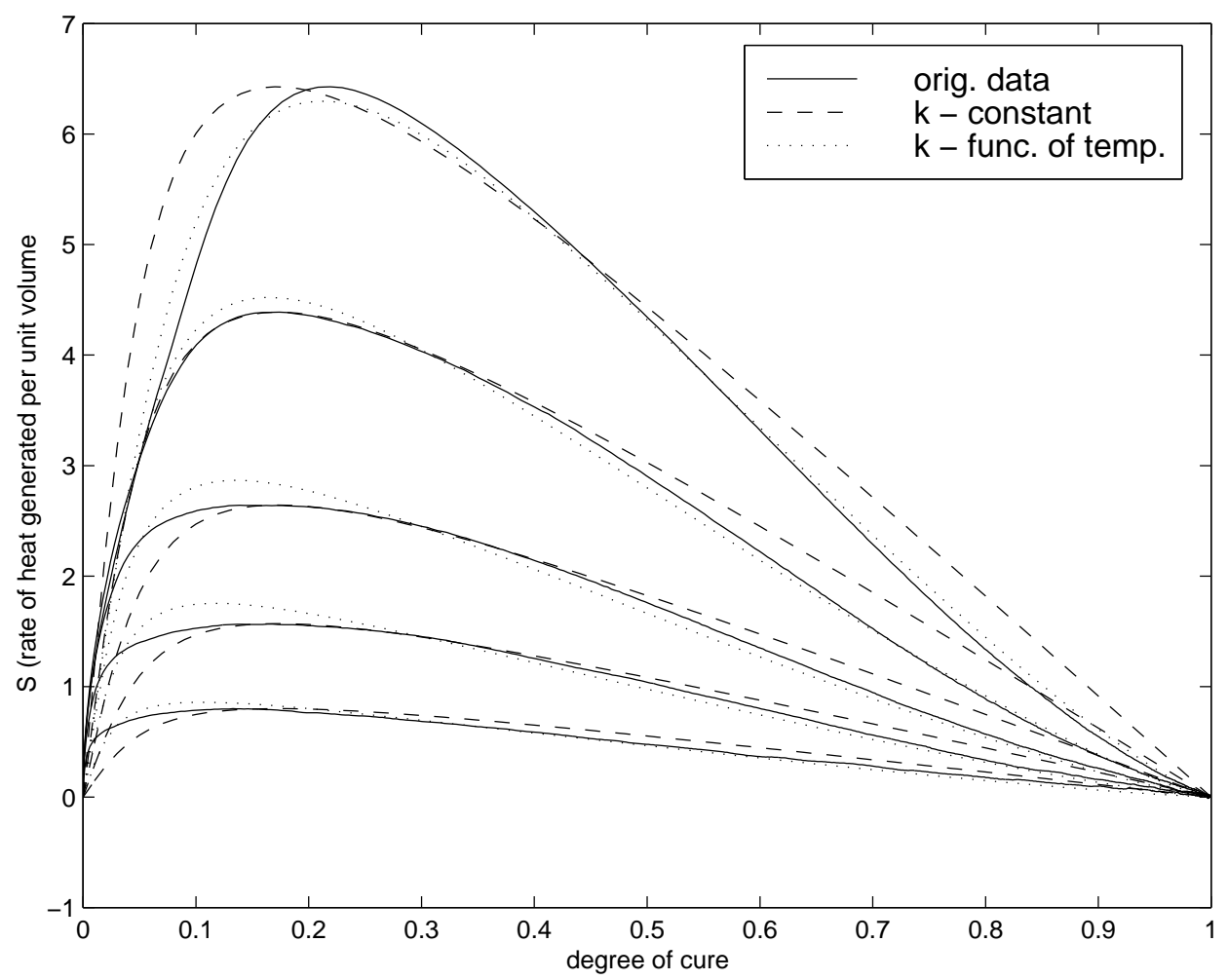

Figure 6: Comparison of two approximations to experimental data.

\subsection{Inclusion of the Exothermic Term in Numerical Simulations}

\subsubsection{Implementation of the Exothermic Term}

We incorporated the exothermic terms given by (44) and (48) into the code to determine what, if any, effect the exothermic reaction had on the curing process of our particular adhesive and also to determine which term was most accurate. We began by studying the effects of adding (44) to the preexisting code. The numerical method requires the solutions to the differential equations:

$$
\frac{d \alpha}{d t}=(1-\alpha) \tan ^{-1}(20 \alpha) \quad \text { and } \quad \frac{d T}{d t}=f(T)
$$

and since neither of these equations can be solved explicitly, we apply backward Euler to advance the time step with Newton iterations to solve the resulting nonlinear equation at each time step. 
These equations are solved as separate steps in the operator splitting method described in Section 3. Clearly this method is more time consuming than using an explicit solution, but the time may be reduced by limiting the number of Newton's iterations to four iterations found to be sufficient for convergence.

Using the above method, we were able to plot the temperature profiles for typical horizontal slices of adhesive. For these simulations, we removed the SMC's from the model so that the behavior of the adhesive alone could be explored. We considered a normalized layer of adhesive with dimensions described by $0 \leq x \leq 1,0 \leq y \leq 1,0 \leq z \leq 1$. To simplify the computations, we assumed that the boundary conditions on the top and bottom were were held constant at $20^{\circ} \mathrm{C}$. Similarly, the initial temperature of the adhesive $\left(T_{0}\right)$ and the ambient temperature $\left(T_{\infty}\right)$ were set to $20^{\circ} \mathrm{C}$. Since our interest here was in the effect of an exothermic reaction, the term $\dot{q}_{r f}$ was set to be 1 , so that any sudden change in heating would be due to the exothermic reaction. We plotted the temperature profiles for the slice of adhesive described by $z=0.1$ for five seconds -see Figure 7 . The resulting profiles were consistent with our expectations based on the known behavior of the adhesive. We noticed that there was a significant increase in temperature in the first two seconds. Since the radio-frequency source term was set equal to one, we concluded that this heating must be due to an internal exothermic reaction. Thus, we demonstrated that failure to include an exothermic source term would result in discrepancies between the simulated data and the actual experimental data. 

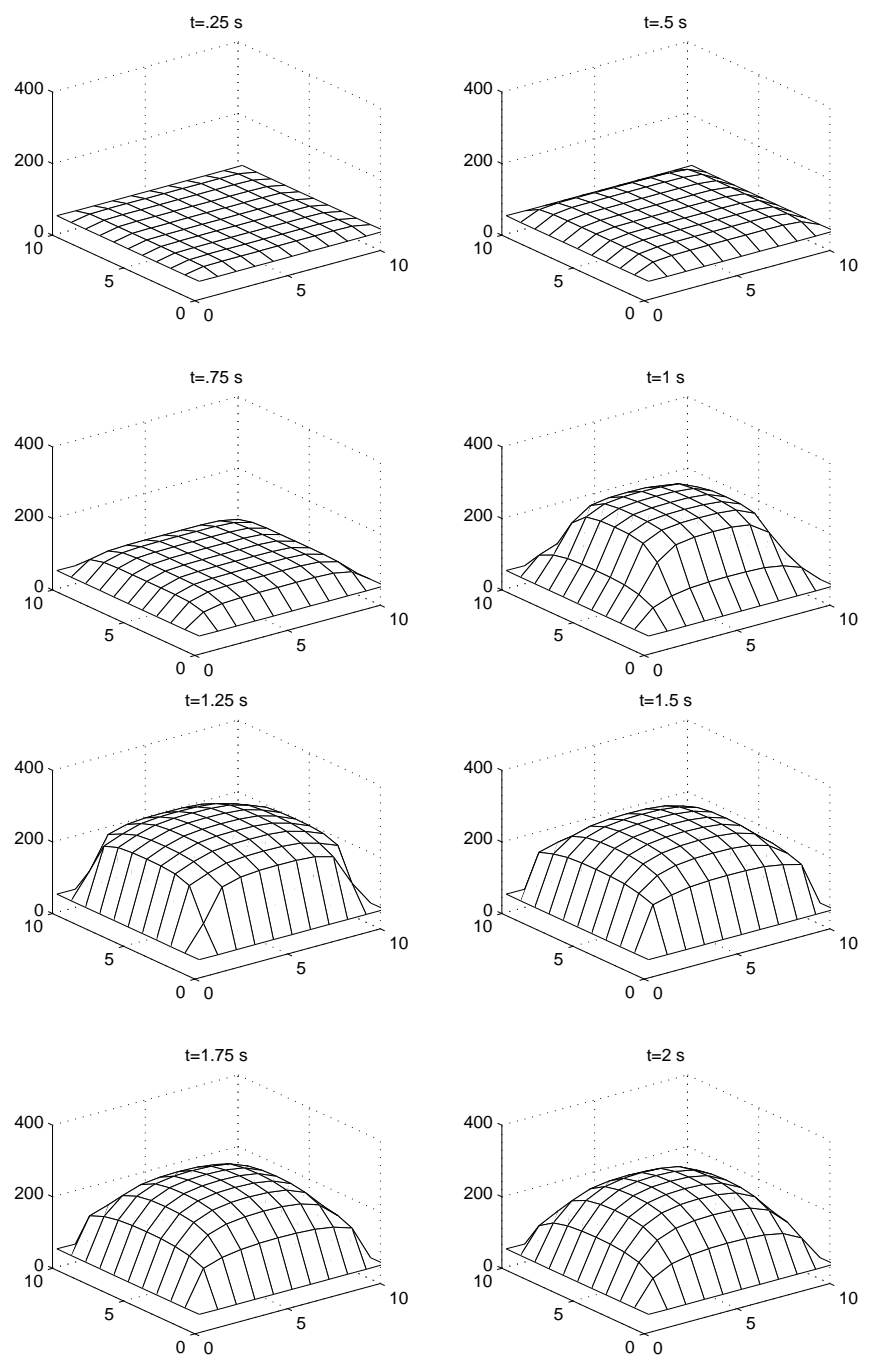

Figure 7: Heat profiles of adhesive slice at $z=.1$ for varying time. 
Recall that we also developed a second form for $S(T, \alpha)$ given by (48) which approximated the experimental data much more closely. We expected to find discrepancies between the simulations described above and the simulations with (48) included. The above method was again used to include (48), with the only addition being that $K(T)$ was first evaluated using the value of $T$ found by the solution of the first equation of (39) at each time step. The boundary and initial conditions were identical to those in the first set of simulations and these simulations yielded results which, when plotted, were virtually indistinguishable from the plots in Figure 7, and hence are not shown here.

\subsubsection{Comparison of the Simulations}

For a more accurate comparison between the simulations with $K=20$ and those with $K=K(T)$, we collected temperature values at the center point of the first slice $(.5, .5, .1)$ and an off-center point $(.2, .2, .1)$ of the same slice at time $t=0.25$ through $t=9$ seconds in increments of 0.25 seconds for the simulations involving the functions given by (44) and (48). The resulting points were plotted against each other for the center and the off-center points -see Figure 8a and Figure 8b.

As can be seen, the profiles were very similar. We used the same method to test points on the center layer $(z=.5)$. This time, the graphs in Figure 9a-b resembled each other even more closely. The code with (44) for the exothermic term produced results which lagged behind those from the code with (48) by approximately 0.25 seconds, but the temperatures reached the same peak value and descended at the same rate. Since there was very little difference in the results, but there was a significant difference in the computational time, we approximated $S(T, \alpha)$ with $\hat{S}(T, \alpha)=f(T)(1-\alpha) \tan ^{-1}(20 \alpha)$ in all subsequent simulations reported on here. 

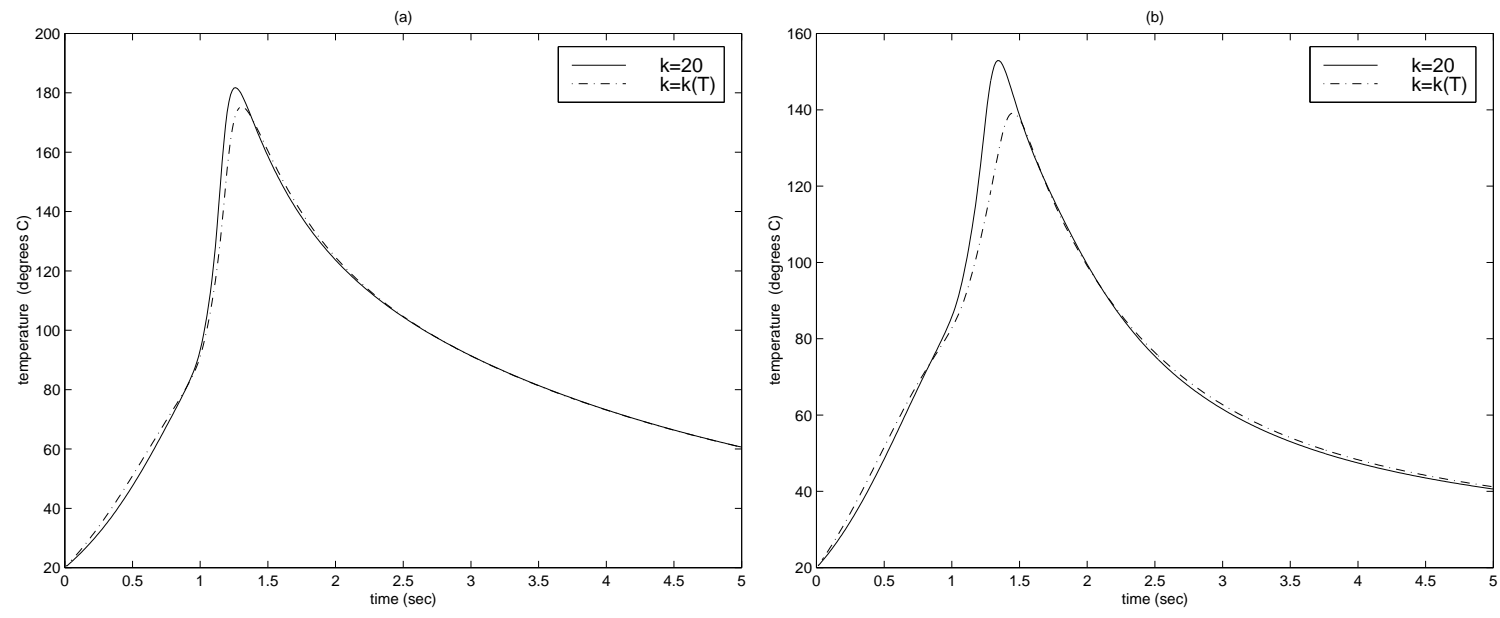

Figure 8: Temperature of adhesive at (a) $(.5, .5, .1)$ and (b) $(.2, .2, .1)$
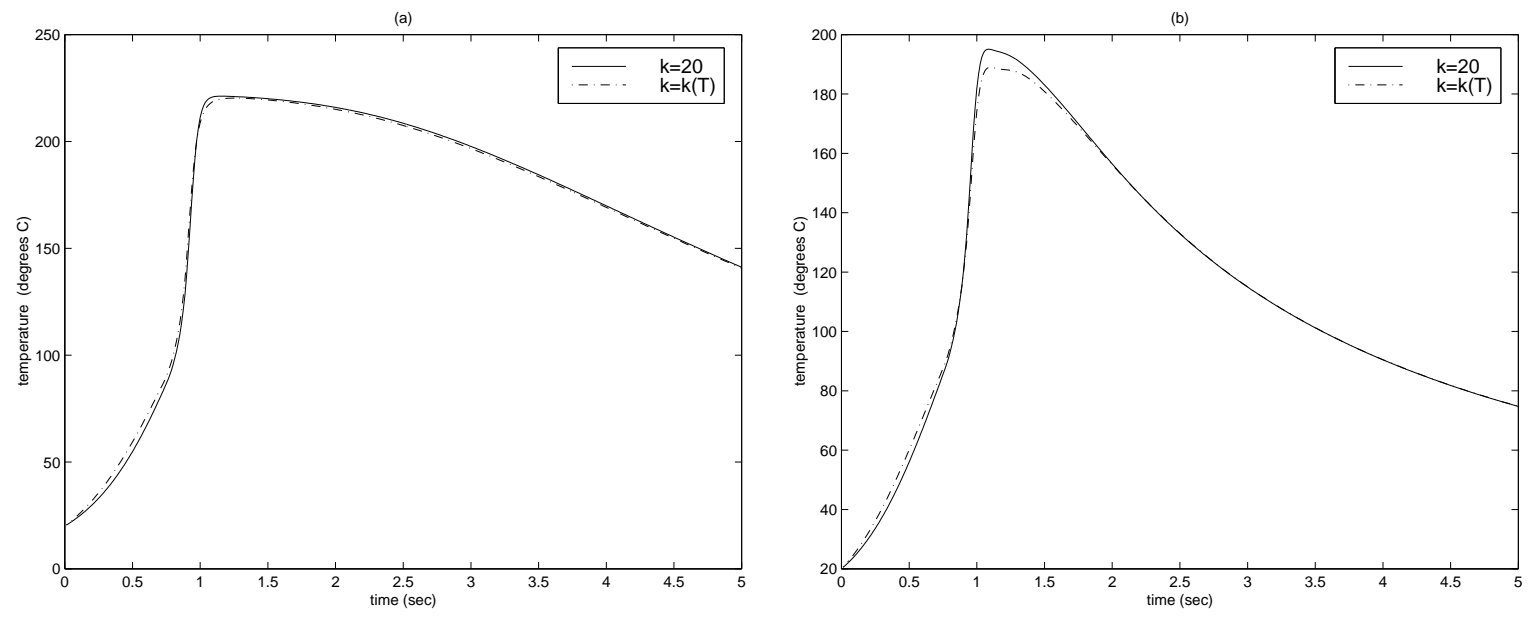

Figure 9: Temperature of center slice of adhesive at (a) $(.5, .5, .5)$ and (b) $(.2, .2, .5)$ 


\subsubsection{Convergence}

Convergence of the results of the code to a solution was tested in order to determine the value of $\Delta t$ which produced the most accurate results. In order to test for convergence, we kept the number of time steps used in each increment constant $(n \max =10)$ and varied the size of the time increment $(\operatorname{tmax})$. We ran the code for $\operatorname{tmax}=.1, .05, .025, .0125, .01, .009$ to obtain temperature values at $(.5, .5, .1)$ between $t=0$ and $t=2$ seconds. The resulting temperature values at that point were plotted against time for each value of tmax in Figure 10. Careful analysis of the data plotted in Figure 10 suggested that the solution began to become unstable when $\Delta t$ was less than .00125 (i.e., $\operatorname{tmax}<.0125)$. Hence, we set the size of the time step $\Delta t=.00125$ for our simulations.

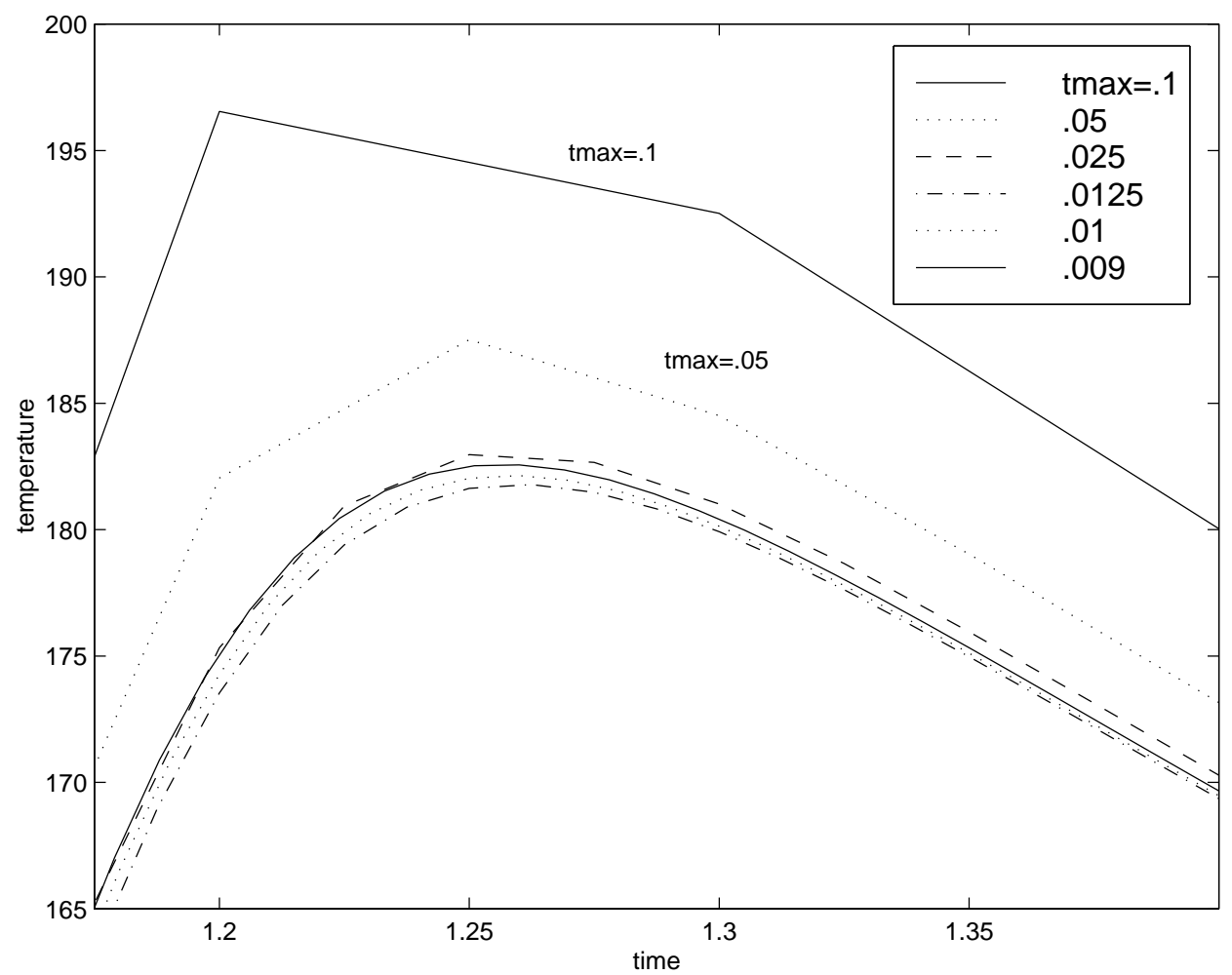

Figure 10: Temperature values of adhesive at $(.5, .5, .1)$ for different values of $\operatorname{tmax}$. 


\subsubsection{Initial Temperature and Exothermic Flash Point}

Finally, we investigated the behavior of the solutions for various initial temperatures $T_{0}$, of the adhesive. We conducted simulations through $t=2$ seconds with a time increment of .0125 and 10 steps in each time increment. The temperatures at spatial locations $(.5, .5, .1)$ and $(.2, .2, .1)$ were tabulated for initial temperatures $T_{0}=10,20,30,40^{\circ} \mathrm{C}$ which are all reasonable temperatures for the adhesive being tested. As expected, for both locations a higher initial temperature of the adhesive resulted in an earlier exothermic reaction. The temperature values remained within a realistic range, although a higher initial temperature resulted in a higher maximum temperature, which was also physically viable.

A sudden increase in temperature in the adhesive, which we termed an exothermic flash point, was discerned in simulations (e.g., see Figure 7 and compare $t=.75 \mathrm{sec}$ with $t=1.0 \mathrm{sec}$ ). Since the flash point can best be seen by looking for a sudden increase in the slope of the temperature versus time curve, we plotted the slopes of the curves with $T_{0}=10,20,30,40^{\circ} \mathrm{C}$ in Figure 11.

An interesting result of these simulations was that that the temperatures at which the exothermic flash point occurred were very close to each other $\left(\approx 85^{\circ} \mathrm{C}\right)$, regardless of the initial temperature. Knowing the approximate temperature at which the flash point occurs regardless of initial temperature will be of importance in future applications when control of the heating of the adhesive becomes an issue. 


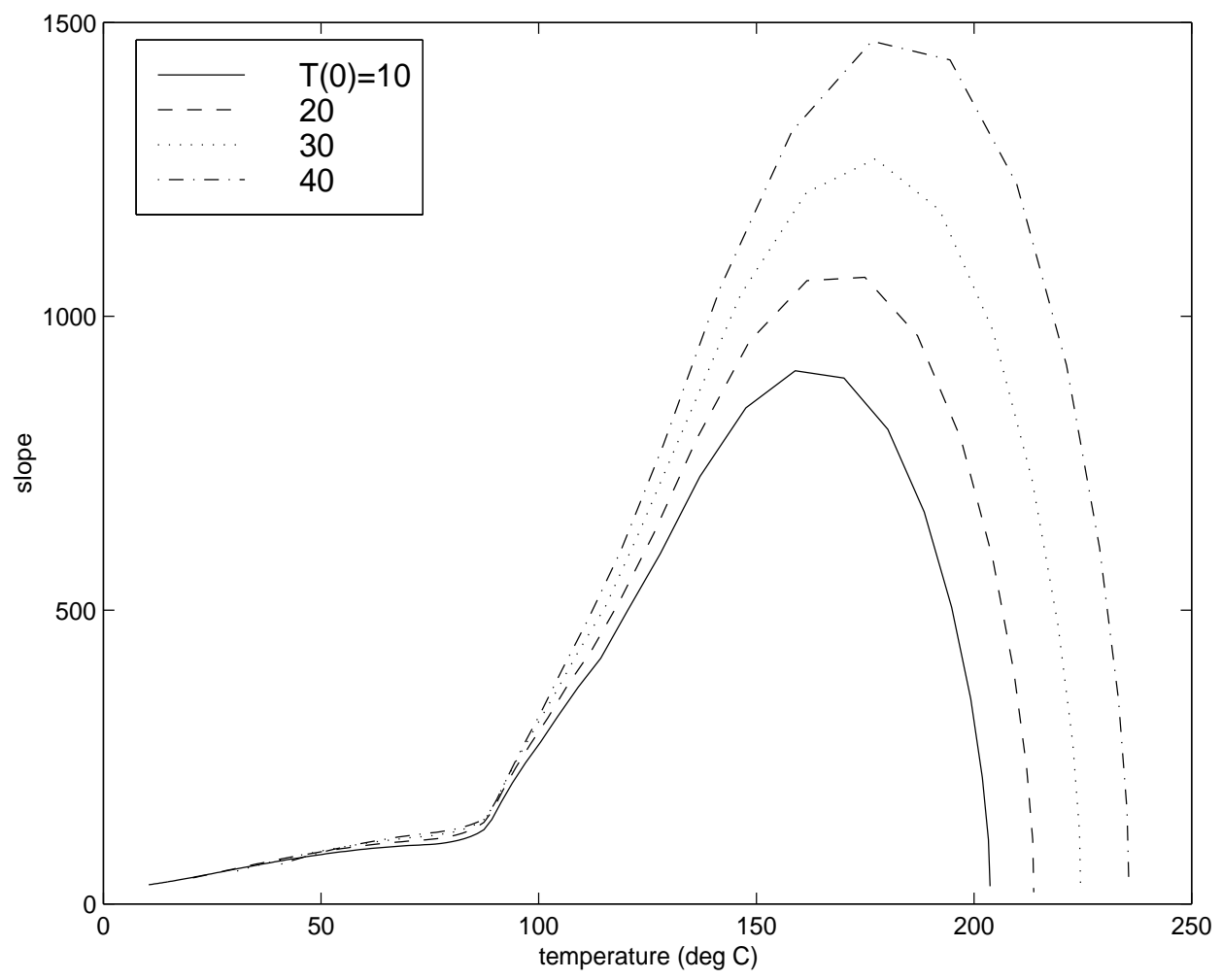

Figure 11: Slope of temperature profiles for different initial temperatures, $T_{0}$, of adhesive.

\section{Summary}

We have derived a model for the radio-frequency curing of adhesives used to bond composites. The model takes into account the heat generated by the conversion of electrical energy to molecular vibrational energy and by an internal exothermic reaction that is part of the curing process. We have described the modeling process used to determine an appropriate form for the term representing the exothermic reaction. We have also proven existence and uniqueness in a constructive manner. thereby obtaining a framework for the numerical method implemented in our investigations. Using experimental data with the computational package, we have shown the importance of including an exothermic term in our model. If such a term is not included the computational simulations can not accurately approximate the heat profiles obtained in physical experiments. 
The model described in this paper is for a specific epoxy adhesive. The exothermic term derived here may not be of an appropriate form to describe other adhesives. In fact, many substances produce no exothermic reaction. It should not be difficult to carry out the experiments described within this paper to obtain data needed to follow the method detailed in Section 4 to determine what, if any, effect the exothermic contribution has on the overall heating of an adhesive. 


\section{A Appendix}

In this appendix we discuss a Bartels-Stewart $[\mathrm{H}]$ method for solving the matrix Liapunov equation (40):

$$
Q \mathbf{T}\left(\mathbf{Q}_{\mathbf{z}}+\Delta t \mathbf{H}_{\mathbf{z}}\right)+\Delta t \mathrm{HT}_{\mathbf{z}}=\mathrm{Q} \hat{\mathbf{T}} \mathbf{Q}_{\mathrm{z}}+\Delta \mathrm{t} \mathbf{F}=\hat{\mathbf{F}}
$$

where $\mathbf{T}=\mathbf{T}^{\mathbf{k}+\frac{1}{2}}$ and $\hat{\mathbf{T}}=\mathbf{T}^{\mathbf{k}}$. Define the symmetric matrix $\tilde{\mathbf{H}}_{\mathbf{z}}=\mathbf{Q}_{\mathbf{z}}^{-\frac{1}{2}} \mathbf{H}_{\mathbf{z}} \mathbf{Q}_{\mathbf{z}}^{-\frac{1}{2}}$ and let $\mathbf{U}_{\mathbf{z}}$ be a real orthogonal matrix such that

$$
\mathbf{U}_{\mathbf{z}}^{\mathrm{t}} \tilde{\mathbf{H}}_{\mathbf{z}} \mathbf{U}_{\mathbf{z}}=\Lambda_{\mathbf{z}}=\operatorname{diag}\left(\left(\lambda_{\mathrm{z}}\right)_{1}, \ldots,\left(\lambda_{\mathrm{z}}\right)_{\mathrm{m}_{3}-1}\right)
$$

where $\left(\lambda_{z}\right)_{l}, 1 \leq l \leq m_{3}-1$ are eigenvalues of $\tilde{\mathbf{H}}_{\mathbf{z}}$. If we define $\tilde{\mathbf{U}}_{\mathbf{z}}=\mathbf{Q}_{\mathbf{z}}^{-\frac{1}{2}} \mathbf{U}_{\mathbf{z}}$ and

$$
\tilde{\mathbf{T}}=\mathbf{T} \mathbf{Q}_{\mathbf{z}}^{\frac{1}{2}} \mathbf{U}_{\mathbf{z}} \quad \text { and } \quad \tilde{\mathbf{F}}=\hat{\mathbf{F}} \tilde{\mathbf{U}}_{\mathbf{z}},
$$

then (49) may be written

$$
Q \tilde{\mathbf{T}}\left(\mathbf{I}+\Delta \mathrm{t} \boldsymbol{\Lambda}_{\mathbf{z}}\right)+\Delta \mathrm{t} H \tilde{\mathbf{T}}=\tilde{\mathbf{F}}
$$

Note that $\left(\tilde{\mathbf{U}}_{\mathbf{z}}\right)^{\mathbf{t}}=\left(\mathbf{Q}_{\mathbf{z}}^{\frac{1}{2}} \mathbf{U}_{\mathbf{z}}\right)^{-1}$. Equation (52) is equivalent to

$$
\left(1+\Delta t\left(\lambda_{z}\right)_{l}\right) Q \tilde{\mathbf{T}}_{\cdot, \mathbf{l}}+\Delta \mathrm{t} H \tilde{\mathbf{T}}_{\cdot, \mathbf{l}}=\tilde{\mathbf{F}}_{\cdot, \mathbf{l}}, \quad 1 \leq 1 \leq \mathrm{m}_{3}-1
$$

or equivalently the system of Liapunov equations

$$
\left(1+\Delta t\left(\lambda_{z}\right)_{l}\right) \mathbf{Q}_{\mathbf{x}} \mathbf{X}_{\mathbf{l}} \mathbf{Q}_{\mathbf{y}}+\Delta t\left(\mathbf{H}_{\mathbf{x}} \mathbf{X}_{\mathbf{l}} \mathbf{Q}_{\mathbf{y}}+\mathbf{Q}_{\mathbf{x}} \mathbf{X}_{\mathbf{l}} \mathbf{H}_{\mathbf{y}}\right)=\mathbf{F}_{\mathbf{l}}, \quad 1 \leq 1 \leq \mathrm{m}_{3}-1
$$

where $\tilde{\mathbf{T}}_{\cdot, \mathbf{l}}=\operatorname{vec}\left(\mathbf{X}_{\mathbf{l}}\right)$ and $\tilde{\mathbf{F}}_{., 1}=\operatorname{vec}\left(\mathbf{F}_{\mathbf{l}}\right)$. Now, we define the symmetric matrices

$$
\tilde{\mathbf{H}}_{\mathbf{x}}=\mathbf{Q}_{\mathbf{x}}^{-\frac{1}{2}} \mathbf{H}_{\mathbf{x}} \mathbf{Q}_{\mathbf{x}}^{-\frac{1}{2}} \text { and } \quad \tilde{\mathbf{H}}_{\mathbf{y}}=\mathbf{Q}_{\mathbf{y}}^{-\frac{1}{2}} \mathbf{H}_{\mathbf{y}} \mathbf{Q}_{\mathbf{y}}^{-\frac{1}{2}}
$$

and let $\mathbf{U}_{\mathbf{x}}$ and $\mathbf{U}_{\mathbf{y}}$ be the diagonalizing real orthogonal matrix of $\tilde{\mathbf{H}}_{\mathbf{x}}$ and $\tilde{\mathbf{H}}_{\mathbf{y}}$, respectively, i.e.,

$$
\mathbf{U}_{\mathbf{x}}^{\mathrm{t}} \tilde{\mathbf{H}}_{\mathbf{x}} \mathbf{U}_{\mathbf{x}}=\Lambda_{\mathbf{x}} \quad \text { and } \quad \mathbf{U}_{\mathbf{y}}^{\mathrm{t}} \tilde{\mathbf{H}}_{\mathbf{y}} \mathbf{U}_{\mathbf{y}}=\Lambda_{\mathbf{y}}
$$


If we define

$$
\tilde{\mathbf{X}}_{\mathbf{l}}=\mathbf{U}_{\mathrm{x}}^{\mathrm{t}} \mathbf{Q}_{\mathrm{x}}^{\frac{1}{2}} \mathbf{X}_{\mathbf{l}} \mathbf{Q}_{\mathbf{y}}^{\frac{1}{2}} \mathbf{U}_{\mathbf{y}} \quad \text { and } \quad \tilde{\mathbf{F}}_{\mathbf{l}}=\tilde{\mathbf{U}}_{\mathrm{x}}^{\mathrm{t}} \mathbf{F}_{\mathbf{l}} \tilde{\mathbf{U}}_{\mathbf{y}}
$$

where $\tilde{\mathbf{U}}_{\mathbf{x}}=\mathbf{Q}_{\mathbf{x}}^{-\frac{1}{2}} \mathbf{U}_{\mathbf{x}}$ and $\tilde{\mathbf{U}}_{\mathbf{y}}=\mathbf{Q}_{\mathbf{y}}^{-\frac{1}{2}} \mathbf{U}_{\mathbf{y}}$, then (53) is equivalent to

$$
\left(1+\Delta t\left(\lambda_{z}\right)_{l}\right) \tilde{\mathbf{X}}_{\mathbf{l}}+\Delta t\left(\boldsymbol{\Lambda}_{\mathbf{x}} \tilde{\mathbf{X}}_{\mathbf{l}}+\tilde{\mathbf{X}}_{\mathbf{l}} \boldsymbol{\Lambda}_{\mathbf{y}}\right)=\tilde{\mathbf{F}}_{\mathbf{l}}, \quad 1 \leq 1 \leq \mathrm{m}_{3}-1
$$

It thus follows from (54)

$$
\left(\tilde{X}_{l}\right)_{i, j}=\frac{1}{1+\Delta t\left(\left(\lambda_{x}\right)_{i}+\left(\lambda_{y}\right)_{j}+\left(\lambda_{z}\right)_{l}\right)}\left(\tilde{F}_{l}\right)_{i, j}
$$

Finally, the solution to (49) is represented by

$$
\mathbf{X}_{\mathbf{l}}=\tilde{\mathbf{U}}_{\mathbf{x}} \tilde{\mathbf{X}}_{\mathbf{l}}\left(\tilde{\mathbf{U}}_{\mathbf{y}}\right)^{\mathrm{t}}, \quad \mathbf{T} \cdot \mathbf{1}=\operatorname{vec}\left(\mathbf{X}_{\mathbf{l}}\right) \text {, and } \mathbf{T}=\tilde{\mathbf{T}}\left(\tilde{\mathbf{U}}_{\mathbf{z}}\right)^{\mathrm{t}}
$$




\section{References}

[BRA] B.R. Baliga, P.L. Rose, and A.M. Ahmed, Thermal modeling of polymerizing polymethylmethacrylate, considering temperature-dependent heat generation, Journal of Biomechanical Engineering, 114 (1992), 251-259.

[BDGJ] H.T. Banks, S.R. Durso, M.A. Goodhart, and M.L. Joyner, On the radio-frequency inputs in dipolar heating of adhesives, Tech. Rep. CRSC-TR98-3, NCSU, January, 1998.

[B] A. Bejan, Heat Transfer, John Wiley \& Sons, New York, 1993.

[CF] P. Constantin and C. Foias, Navier-Stokes Equations, University of Chicago Press, Chicago, 1988.

[H] S.J. Hammarling, Numerical solution of the stable, non-negative definite Lyapunov equation, in Numerical Linear Algebra Techniques for Systems and Control, (R.V. Patel, et. al., eds.), IEEE Press, New York, 1993, 500-516.

[ID] F.P. Incropera and D.P. Dewitt, Fundamentals of Heat and Mass Transfer, John Wiley \& Sons, New York, 1985.

[LM] P.L. Lions and B. Mercier, Splitting algorithms for the sum of two nonlinear operators, SIAM J. Numer. Anal., 16 (1979), 964-979.

[M] G.W. Malaczynski, Adhesive processing by electromagnetic irradiation, Polymer Engineering and Science, 28 (1988), 1270-1274.

[MC] G.W. Malaczynski and G.J. Cinpinski, Selection of adhesive system for radio-frequency heating of structural sheet molding compound components, Polymer Engineering and Science, 36 (1996), 106-116. 
[W] J. Wloka, Partial Differential Equations, Cambridge University Press, Cambridge, 1992.

[Y] N.N. Yanenko, The Method of Fractional Steps, Springer-Verlag, New York, 1971. 